TRANSACTIONS OF THE

AMERICAN MATHEMATICAL SOCIETY

Volume 300, Number 2, April 1987

\title{
POLYNOMIAL ALGEBRAS HAVE POLYNOMIAL GROWTH
}

\author{
DAVID R. FINSTON
}

\begin{abstract}
The definitions and basic properties of Gelfand-Kirillov dimension are extended to algebras over a field which are not necessarily associative. The results are applied to the algebra of polynomial functions on an arbitrary finite dimensional algebra to obtain polynomial growth (i.e. integral G-K dimension) for these algebras. The G-K dimension of the polynomial algebra in one indeterminate is shown to be constant on the category of all finite dimensional nomial extensions of an associative algebra.
\end{abstract}

I. Introduction. The algebra of polynomial functions as an arbitrary algebra was introduced in [10] where an elegant generalization of the fundamental theorem of algebra was obtained for polynomial functions in one indeterminate over an arbitrary finite dimensional algebra. This structure was further investigated in [4] where it was shown that a certain amount of classical Galois theory over fields could be extended to arbitrary finite dimensional algebras.

As is the case in the category of commutative, associative, unital $R$-algebras, the algebra of polynomial functions on a nonassociative algebra is a free object in a naturally arising category of, not necessarily associative, algebras (namely the category of algebras with nomial homomorphisms $[3,11])$. In case the algebra is a commutative field, its algebra of polynomial functions on a set $X$ agrees with the usual polynomial ring and it is well known [1] that its Gelfand Kirillov dimension (henceforth G-K dimension) is equal to the common value of the transcendence degree of the rational function field and Krull dimension. It is, therefore, natural to investigate the G-K dimension of algebras of polynomial functions. To that end, the definitions and basic properties of G-K dimension have been adapted to handle nonassociativity.

§II describes the extension of G-K dimension to nonassociative algebras. The treatment follows [1], and the reader is referred there or to [8, Chapters 1-4], for complete proofs in the associative case.

A great deal of information regarding the structure of an algebra can be recovered from its multiplication algebra, and the structure of the algebra as a faithful left module for its multiplication algebra [5]. The G-K dimension of an algebra computed as a module for its multiplication algebra is discussed in §III.

The algebra of polynomial functions on an algebra $A$ in a set $X$ is described in $\S I V$, in order to keep the exposition as self-contained as possible. Some new technical results, needed for the computation of the G-K dimensions are presented.

Received by the editors January 31, 1986.

1980 Mathematics Subject Classification (1985 Revision). Primary 17A99.

(C)1987 American Mathematical Society $0002-9947 / 87 \$ 1.00+\$ .25$ per page 
The structure theory of finite dimensional associative algebras carries over nicely to the algebra of polynomial functions when the base algebra is associative, allowing for a relatively easy proof of polynomial growth. The proof of the special case is included in $\S \mathrm{V}$ since it is similar to, but less technically obscure than, the general argument. Moreover, the proof for the associative case allows for the proof, in §VII, that $\operatorname{Dim} A\langle x\rangle=\operatorname{Dim} B\langle x\rangle$ for all nomial extensions $B$ of the associative algebra $A$. Finally, the polynomial algebra of an associative algebra and its homomorphic images provide a source of infinite dimensional examples of associative algebras whose structures are strongly controlled by that of the finite dimensional base algebra.

$\S$ VI contains the proof that for arbitrary finite dimensional $A$ and finite set $X$, $\operatorname{Dim} A\langle X\rangle$ is a natural number.

$\S$ VII introduces the category of algebras with nomial morphisms. It is proved there that $\operatorname{Dim}-(x)$ is an invariant on the category of finite dimensional nomial extensions of an associative algebra. It is conjectured that the result holds for arbitrary finite dimensional algebras.

Certain foundational results on the algebra of polynomial functions and nomial homomorphisms first appeared in [11]. Since that source may be difficult to obtain, reference is also made to [3] for further details about these results. §§II-VI were part of the author's dissertation written under the supervision of Professor Helmut Röhrl at the University of California, San Diego. The author wishes to thank Professor Röhrl for his guidance in that effort and for his continued invaluable assistance. The author wishes also to thank Professor Adrian Wadsworth for his careful reading of a preliminary version and his many valuable criticisms.

II. Preliminaries on G-K dimension. Let $k$ be an infinite field. A $k$-algebra $A$ is a $k$-vector space together with a vector space homomorphism $\mu: A \otimes_{k} A \rightarrow A$ giving the multiplication. The $\mu$ will be suppressed throughout, and $\mu(a \otimes b)$ will be denoted $a b$, except for the following convention. If $a_{1}, a_{2}, \ldots, a_{n} \in A$ and $\beta(n)$ is a bracketting of $n$ symbols, the result of multiplying $a_{1}, \ldots, a_{n}$ according to $\beta(n)$ will be denoted $\mu_{\beta(n)}\left(a_{1}, \ldots, a_{n}\right)$. If $X$ is a set, $k\{X\}$ will denote the free nonassociative algebra on $X$. A $k$-algebra will be called finite type if it is a homomorphic image of $k\{X\}$ for some finite set $X$.

Given a finite type $k$-algebra $A$, a filtration of $A$ by finite dimensional subspaces $V_{n}$ is constructed as follows: let $V$ be a finite dimensional subspace of $A$ containing a set of algebra generators. Set $V^{1}=V$ and $V^{i}=\operatorname{span}_{k}\left\{\mu_{\beta(i)}\left(v_{1}, v_{2}, \ldots, v_{i}\right) \mid v_{j} \in V\right.$, $\beta(i)$ runs through all possible brackettings of $i$ symbols $\}$. Set $V_{n}=\sum_{1 \leqslant i \leqslant n} V^{i}$. Clearly, $A=\cup_{n \geqslant 1} V_{n}$, and $n \mapsto d_{V}(n)=\operatorname{dim}_{k} V_{n}$ is a monotone nondecreasing function on the natural numbers $\mathbf{N}$.

The ordering on $\mathscr{F}=\{f: \mathbf{N} \rightarrow \mathbf{N} \mid f$ is nondecreasing $\}$, given by $f \leqslant g$ iff there is an $m \in \mathbf{N}$ with $f(u) \leqslant g(m n)$ for all $n$, induces an equivalence relation $\sim$ on $\mathscr{F}$ given by $f \sim g$ iff $f \leqslant g$ and $g \leqslant f$. The equivalence class of $f$ is denoted $w(f)$ and is called the growth of $f$. A routine argument [1, Satz 1.6] shows that $w\left(d_{V}\right)=w\left(d_{W}\right)$ for any pair of finite dimensional generating subspaces $V$ and $W$ of $A$. Thus the growth of $A$, denoted $w(A)$, is well defined by $w\left(d_{V}\right)$. 
(2.1) Definition. (1) $\operatorname{Dim} A=\lim \sup \log _{n} d_{V}(n)$ is the $G$ - $K$ dimension of $A$.

(2) DIM $A=\limsup \log _{n} \ln \left(d_{V}(n)\right)$ is the superdimension of $A$.

For arbitrary $A$ the $G-K$ dimension and superdimension are

(3) $\operatorname{Dim} A=\sup \{\operatorname{Dim} B \mid B$ is a finite type subalgebra of $A\}$,

(4) $\operatorname{DIM} A=\sup \{\mathrm{DIM} B \mid B$ is a finite type subalgebra of $A\}$.

Certain of the estimates on G-K dimension and superdimension proved in [1, paragraph 2] which carry over to arbitrary algebras can be obtained by considering the number of ways to bracket $n$ symbols. An application of Stirling's formula yields that this number $C_{n}=(2 n-1)^{-1}\left(\begin{array}{c}2 n-1 \\ n\end{array}\right)$, the $n$th Catalan number, is bounded by $k^{n} /(2 n-1)^{3 / 2}$, where $k$ is a constant independent of $n$.

(2.2) Proposition. (1) $\operatorname{Dim} A=0$ if every finite type subalgebra of $A$ is finite dimensional.

(2) $w(A) \leqslant w\left(n \rightarrow e^{n}\right)$.

(3) $0 \leqslant \operatorname{DIM} A \leqslant 1$.

Proof. For (1) it suffices to consider $A$ finite type. If $A$ is finite dimensional, then for some $n, V_{n}=V_{n+1}$ for all $i$, which shows $\operatorname{Dim}(A)=0$.

$$
\bigoplus_{i=1}^{n}\left(\bigoplus_{j=1}^{C_{i}} V \otimes \underset{i \text { times }}{\ldots} \otimes V\right) \rightarrow V_{n},
$$

so that

$$
\begin{aligned}
d_{V}(n) & \leqslant \sum_{i=1}^{n} C_{i} \cdot\left[d_{V}(1)\right]^{i} \\
& \leqslant \sum_{i=1}^{n} \frac{\left[k d_{V}(1)\right]^{i}}{(2 i-1)^{3 / 2}} \leqslant \tilde{K}^{(n+1)}
\end{aligned}
$$

where $\tilde{K}$ is a constant independent of $n$.

(3) follows immediately from (2).

For associative algebras, the converse to assertion (1) is also true. In contrast is the following nonassociative algebra, generated by a single element which is infinite dimensional with G-K dimension equal to zero. Let $A=k\{x\}$, the free nonassociative algebra on the generator $x$, and $I$ the ideal in $A$ generated by $\left\{\mu_{\beta(i)}(x) \mu_{\beta(j)}(x) \mid i \neq j\right\}$. The following sequence of elements of $A: x^{2^{0}}=x$, $x^{2}=x \cdot x, \quad x^{2^{i}}=\left(x^{2^{i-1}}\right)\left(x^{2^{i-1}}\right)$ survive modulo $I$ and in fact $A / I \cong \bigoplus_{i=0}^{\infty} k \bar{x}^{2^{i}}$, where $\bar{a}$ denotes the image of $a \in A$ in $A / I$. $A / I$ is generated by $V=k \bar{x}$, and

$$
V^{i}= \begin{cases}k \bar{x}^{2^{j}}, & i=2^{j}, \\ (0) & \text { otherwise. }\end{cases}
$$

Thus $d_{V}(n) \leqslant 1+\log _{2} n$ for all $n$, and

$$
\operatorname{Dim} A \leqslant \limsup \frac{\ln \left(1+\log _{2}(n)\right)}{\ln n}=0 .
$$


The bounds in (3) are sharp as the free associative, nonassociative, and Lie algebras on two or more generators all have superdimension equal to one. For example, let $A$ be the free Lie algebra on generators $x$ and $y$.

$$
\begin{aligned}
V & =k x+k y, \\
V^{2} & =\operatorname{span}_{k}\{x y\}, \\
V^{3} & =\operatorname{span}_{k}\{(x y) x,(x y) y\} .
\end{aligned}
$$

Using the Lie identities one shows that

$$
V^{n}=\operatorname{span}_{k}\left\{\left(\left(\cdots(x y) a_{3} a_{4}\right) \cdots\right) a_{n} \mid a_{i} \in\{x, y\}\right\},
$$

whence, for $n \geqslant 2, d_{V}(n)=2^{n-2}+2^{n-3}+\cdots+1+2=2^{n-1}+1$. That DIM $A$ $=1$ follows easily.

(2.3) Definition. $w(A)$ is called limiting if $\lim _{n \rightarrow \infty} \log _{n} d_{v}(n)$ exists for every finite dimensional subspace $V$ of $A$.

The elementary properties of G-K dimension in [1, Lemma 3.1] which carry over easily to arbitrary algebras are included in

(2.4) Proposition. Let $A$ and $B$ be nonzero algebras over the field $k$.

(1) $\operatorname{Dim} A \Pi B=\max \{\operatorname{Dim} A, \operatorname{Dim} B\}$.

(2) $\operatorname{Max}\{\operatorname{Dim} A, \operatorname{Dim} B\} \leqslant \operatorname{Dim} A \otimes_{k} B \leqslant \operatorname{Dim} A+\operatorname{Dim} B$.

$\operatorname{Dim} A \otimes_{k} B=\operatorname{Dim} A+\operatorname{Dim} B$ if both $A^{2}=A$ and $B^{2}=B$ and $w(A)$ or $w(B)$ is limiting.

(3) $\operatorname{Dim} C \leqslant \operatorname{Dim} A$ for all quotients and subalgebras $C$ of $A$.

(4) $\operatorname{Dim} A / I_{I} \cap \cdots \cap I_{r}=\max _{1 \leqslant j \leqslant r}\left\{\operatorname{Dim} A / I_{j}\right\}$ for ideals $I_{1}, \ldots, I_{r}$ of $A$.

(5) If $R$ is a commutative, associative, unital k-algebra, and $A$ has the structure of an $R$-algebra as well, then for any multiplicative set $S \subset R$ with $0 \notin S, \operatorname{Dim} A=$ $\operatorname{Dim}\left(S^{-1} R \otimes A\right)$.

Proof. Except for (2) the proofs are routine and identical to those given in [1, Lemma 3.1].

For (2), let $V$ (resp. $W$ ) be a finite dimensional generating subspace for $A$ (resp. $B$ ) and $X \subset A \otimes_{k} B$ a finite dimensional subspace. Then $X \subset V_{n} \otimes W_{n}$ for some $n$, so that

$$
X^{m} \subset\left(V_{n} \otimes W_{n}\right)^{m} \subset V_{n m} \otimes W_{n m} \quad \text { and } \quad X_{m} \subset V_{n m} \otimes W_{n m} \quad \text { for all } m .
$$

By taking the supremum over finite type subalgebras of $A \otimes_{k} B, \operatorname{Dim}\left(A \otimes_{k} B\right) \leqslant$ $\operatorname{Dim} A+\operatorname{Dim} B$ follows.

With $V$ and $W$ as above, $V \otimes W$ generates a subalgebra of $A \otimes B$. Clearly,

$$
\operatorname{dim}_{k}\left(V \otimes W+(V \otimes W)^{2}+\cdots+(V \otimes W)^{n}\right) \geqslant \operatorname{Max}\left\{d_{V}(n), d_{W}(n)\right\},
$$

from which $\operatorname{Dim} A \otimes B \geqslant \operatorname{Max}\{\operatorname{Dim} A, \operatorname{Dim} B\}$ follows.

Assume that $A^{2}=A, B^{2}=B$, and let $V^{\prime}$ and $W^{\prime}$ be finite dimensional generating subspaces of $A$ and $B$ respectively. Since $A^{2}=A$,

$$
V^{\prime} \subset\left(V^{\prime}\right)^{2}+\left(V^{\prime}\right)^{3}+\cdots+\left(V^{\prime}\right)^{n} \text { for some } n \text {. }
$$


Set $V=\sum_{i=1}^{n}\left(V^{\prime}\right)^{i}$; then $V \subset V^{2}$. Similarly, let $W$ be a finite dimensional generating subspace for $B$ with $W \subset W^{2}$. Note that $V_{n}=V^{n}$ and $W_{n}=W^{n}$ for all $n$. Let $U=V^{2} \otimes W^{2}$ and observe that $V^{n} \otimes W^{n} \subset U^{n}$ for all $n$. To see this, we can reduce this, by induction, to showing that for $v \in V$ and $w_{1}, \ldots, w_{n} \in W, v \otimes$ $\mu_{\beta(n)}\left(w_{1}, \ldots, w_{n}\right) \in U^{n}$, and for $v_{1}, \ldots, v_{n} \in V, w \in W, \mu_{\beta(n)}\left(v_{1}, \ldots, v_{n}\right) \otimes w \in U^{n}$. We can write

$$
\mu_{\beta(n)}\left(w_{1}, \ldots, w_{n}\right)=\mu_{\beta(l)}\left(w_{1}, \ldots, w_{l}\right) \mu_{\beta(n-l)}\left(w_{l+1}, \ldots, w_{n}\right),
$$

and $v=\sum \alpha_{i} v_{i} v_{i}^{\prime}$ where $v_{i}, v_{i}^{\prime} \in V$ and $\alpha_{i} \in k$. Thus

$$
\begin{aligned}
& v \otimes \mu_{\beta(n)}\left(w_{1}, \ldots, w_{n}\right)=\sum \alpha_{i} v_{i} v_{j}^{\prime} \otimes \mu_{\beta(l)}\left(w_{1}, \ldots, w_{l}\right) \mu_{\beta(n-l)}\left(w_{l+1}, \ldots, w_{n}\right) \\
& \quad=\sum_{i}\left[\alpha_{i} v_{i} \otimes \mu_{\beta(l)}\left(w_{1}, \ldots, w_{l}\right)\right]\left[v_{j}^{\prime} \otimes \mu_{\beta(n-l)}\left(w_{l+1}, \ldots, w_{n}\right)\right] \in U^{l} U^{n-l} \subset U^{n} .
\end{aligned}
$$

The identical argument shows $\mu_{\beta(n)}\left(v_{1}, \ldots, v_{n}\right) \otimes w \in U^{n}$. Now $V^{n} \otimes W^{n} \subset U^{n}$ implies

$$
\begin{aligned}
\operatorname{Dim} A \otimes B & \geqslant \limsup \log _{n}\left(d_{V}(n) \cdot d_{W}(n)\right) \\
& =\lim \sup \left[\log _{n} d_{V}(n)+\log _{n} d_{W}(n)\right] .
\end{aligned}
$$

If either $A$ or $B$ is limiting,

$$
\begin{aligned}
\limsup & {\left[\log _{n} d_{V}(n)+\log _{n} d_{W}(n)\right] } \\
= & \limsup \log _{n} d_{V}(n)+\lim \sup \log _{n} d_{W}(n),
\end{aligned}
$$

and $\operatorname{Dim} A \otimes B=\operatorname{Dim} A+\operatorname{Dim} B$.

The following lemma provides some information on the behavior of G-K dimension under restriction and extension of scalars.

(2.5) LeMmA. Let $A$ be a $k$-algebra and $L$ a finite extension field of $k$.

(1) If $A$ has the structure of an L-algebra then $\operatorname{Dim}_{L} A=\operatorname{Dim} A$.

(2) $\operatorname{Dim}_{L}\left(L \otimes_{k} A\right)=\operatorname{Dim} A$.

Proof. (1) If $V$ is any finite dimensional $L$ subspace of $A$ then $\operatorname{dim}_{k} V=$ $[L: k] \operatorname{dim}_{L} V$. In particular, $\log _{n} \operatorname{dim}_{k} V_{n}=\log _{n}[L: k]+\log _{n} \operatorname{dim}_{L} V_{n}$, taking limits superior, we obtain $\operatorname{Dim} A=\operatorname{Dim}_{L} A$.

(2) If $V$ is a finite dimensional $k$ subspace of $A$ then $L \otimes_{k} V$ is a finite dimensional subspace of $L \otimes_{k} A$, and $\operatorname{dim}_{L}\left(L \otimes_{k} V\right)=\operatorname{dim}_{k} V$. If $B$ is a finite type subalgebra of $L \otimes_{k} A$, we can enlarge $B$, if necessary, to obtain $B^{\prime} \cong L \otimes B_{0}$ for some finite type subalgebra $B_{0}$ of $A$. Now choose a basis for a finite dimensional generating subspace $U$ of $B^{\prime}$ of the form $\left\{1 \otimes v_{i}\right\}$ where $\left\{v_{i}\right\}$ is a $k$ basis for a generating subspace of $B_{0}$. Then $U_{n} \cong L \otimes\left(U_{n} \cap A\right) \cong L \otimes(U \cap A)_{n}$, where $U \cap A$ (resp. $U_{n} \cap A$ ) denotes the intersection of $U$ (resp. $U_{n}$ ) with the image of $A$ in $L \otimes A$.

III. The multiplication algebra. The multiplication algebra $\mathscr{M}_{R}(A)$ of an $R$-algebra $A$ is the subalgebra of $\operatorname{End}_{R} A$ generated by the identity and all right and left multiplications by elements of $A$ considered as $R$-endomorphisms of $A$. The multiplication ideal $\mathscr{M}_{R}^{*}(A)$ is the $R$ subalgebra of $\mathscr{M}_{R}(A)$ generated by the right 
and left multiplications by elements of $A$. Multiplication algebras were investigated in [5] and we make frequent use of the following result from that paper:

(3.1) LEMMA. Let $A$ be an $R$-algebra finitely generated projective as an $R$ module and $S$ a commutative associative unital R-algebra. Then $\mathscr{M}_{S}\left(S \otimes_{R} A\right) \cong S \otimes_{R} \mathscr{M}_{R}(A)$.

(3.1)' LEMMA. If $k$ is a field and $A$ a possibly infinite dimensional $k$-algebra, then $L \otimes_{k} \mathscr{M}(A) \cong \mathscr{M}_{L}\left(L \otimes_{R} A\right)$ for any commutative associative unital $k$-algebra $L$.

Proof. If $\left\{e^{i} \mid i \in I\right\}$ is a $k$ basis for $A$, then $\mathscr{M}(A)$ is generated as an algebra by $\left\{\lambda_{e^{i}}, \rho_{e^{i}} \mid i \in I\right\} \cup\left\{\mathrm{id}_{A}\right\} . \mathscr{M}\left(L \otimes_{k} A\right)$ is generated by $\left\{1 \otimes \lambda_{e^{i}}, 1 \otimes \rho_{e^{i}}\right\} \cup\{1 \otimes$ $\left.\operatorname{id}_{A}\right\}$, since $\left\{1 \otimes e^{i} \mid i \in I\right\}$ constitutes an $L$ basis for $L \otimes A$.

Clearly, $A$ has the structure of a left $\mathscr{M}(A)$-module, and hence a Gelfand Kirillov dimension as a module for $\mathscr{M}(A)$. The definition of the $\mathrm{G}-\mathrm{K}$ dimension of a module is included to make the exposition self-contained. More details can be found in [7 or 8, Chapter 5].

Let $A$ be a finite type, associative unital $k$-algebra and $M$ a finitely generated left $A$-module. Let $V$ be a finite dimensional generating subspace of $A$ and $U$ a finite dimensional subspace of $M$ generating $M$ as a left $A$-module. Construct a filtration of $M$ by finite dimensional subspaces $U_{i}$ as follows:

$$
\begin{aligned}
& U_{0}=U, \\
& U_{n}=V_{n} U, \text { where } V_{n}=\sum_{i \leqslant n} V^{i} \text { is a filtration of } A .
\end{aligned}
$$

Clearly, the function $d_{V}^{\prime}(n)=\operatorname{dim}_{k}\left(U_{n}\right)$ is monotone nondecreasing on the natural numbers, and a routine argument shows that its growth is independent of both $U$ and $V$.

(3.2) Definition. (1) If $A$ is a finite type associative unital $k$-algebra and $M a$ finitely generated left $A$-module, the $G-K$ dimension of $M$, denoted $\operatorname{Dim}_{A} M$, is $w\left(d_{V}^{\prime}(n)\right)$.

(2) If $A$ is as above and $M$ is arbitrary, $\operatorname{Dim}_{A} M=\sup \left\{\operatorname{Dim}_{A} N \mid N\right.$ is a finitely generated submodule of $M$ \}.

(3) If $A$ and $M$ are arbitrary $\operatorname{Dim}_{A} M=\sup \left\{\operatorname{Dim}_{A^{\prime}} M \mid A^{\prime}\right.$ is a finite type subalgebra of $A$ \}.

Most of the following proposition appears in [7].

(3.3) Proposition. Let $A$ be an associative, unital, finite type k-algebra and $M a$ finitely generated left $A$-module, then

(1) If $M=M_{1}+\cdots+M_{n}$, a sum of left $A$-submodules, then $\operatorname{Dim}_{A} M=$ $\operatorname{Max}_{i}\left\{\operatorname{Dim}_{A} M_{i}\right\}$

(2) $\operatorname{Dim}_{A} M \leqslant \operatorname{Dim} A$.

(3) $\operatorname{Dim}_{A} N \leqslant \operatorname{Dim}_{A} M$ for all quotients and submodules $N$ of $M$.

(4) If $I$ is an ideal of $A$ contained in the annihilator of $M$, then $\operatorname{Dim}_{A / I}(M)=$ $\operatorname{Dim}_{A} M$.

(5) If there is an element $m \in M$ with $\operatorname{Ann}_{A}(m)=(0)$, then $\operatorname{Dim}_{A} M=\operatorname{Dim} A$.

(6) If $m_{1}, \ldots, m_{n} \in M$ satisfy $\bigcap_{i} \operatorname{Ann}_{A}\left(m_{i}\right)=(0)$, then $\operatorname{Dim}_{A} M=\operatorname{Dim} A$. 
Proof. For (1)-(4) see [7].

(5) Suppose $\operatorname{Ann}_{A}(m)=(0)$. Let $U$ be a finite dimensional subspace of $M$ containing $m$ and a set of left module generators, and $V$ a subspace of $A$. From $U_{r}=V_{r} U \supset V_{r} m \cong V_{r}$ as vector spaces we obtain $d_{V}^{\prime}(r) \geqslant d_{V}(r)$. This inequality together with (2) proves the assertion.

(6) The element $\left(m_{1}, \ldots, m_{n}\right) \in \oplus_{1}^{n} M$ satisfies $\operatorname{Ann}_{A}\left(\left(m_{1}, \ldots, m_{n}\right)\right)=$ $\bigcap_{i} \operatorname{Ann}_{A}\left(m_{i}\right)=(0)$. Parts (1) and (5) prove the assertion.

Any algebra $A$ is a faithful left module over its multiplication algebra. Moreover, if $A$ is finite type, then algebra generators, $a_{1}, \ldots, a_{n} \in A$, serve as left $\mathscr{M}(A)$ module generators: any monomial $M\left(a_{1}, \ldots, a_{n}\right)$ in which $a_{1}$ appears is certainly in the $\mathscr{M}(A)$ submodule generated by $a_{1}$.

(3.4) Lemma. Let $A$ be a (not necessarily associative) finite type $k$-algebra. Then $\operatorname{Dim} A=\operatorname{Dim}_{\mathscr{M}(A)} A$.

Proof. Let $U \subset A$ be a finite dimensional generating subspace with basis $u_{1}, \ldots, u_{m}$. Let $V \subset \mathscr{M}(A)$ be a finite dimensional subspace containing $\mathrm{id}_{A}$ and all right and left multiplications by the $u_{i}$.

For any $s>0, \mu_{\beta(s)}\left(u_{1}, \ldots, u_{s}\right) \in V^{s-1} U$ from which $U_{s} \subset V_{s} U$ follows, proving $\operatorname{Dim} A \leqslant \operatorname{Dim}_{M(A)} A$.

Let $v_{1}, \ldots, v_{m}$ be a basis for $V$. Each $v_{i}$ is a sum of products of left and right multiplications by elements from various $U_{i}$. In particular, $V U \subset U_{n+1}$ for sufficiently large $n$, and in fact $V^{i} \subset U_{n i+1}$ for all $i$. Since id ${ }_{A} \in V$, we have $V^{i} U=V_{i} U$ $\subset U_{n i+1}$, and $d_{V}(i) \leqslant d_{U}((n+1) i)$. This shows $\left(d_{V}^{\prime}\right) \leqslant\left(d_{U}\right)$ from which $\operatorname{Dim}_{M(A)}(A) \leqslant \operatorname{Dim} A$ follows.

If $A$ is a graded (nonassociative) algebra, $\mathscr{M}(A)$ inherits the grading in a natural way. Let $A=\oplus_{-\infty}^{\infty} A_{n}$ where $A_{n}$ is the space of degree $n$ homogeneous elements. Denoting the operator of left (resp. right) multiplication by $a \in A$ by $\lambda_{a}$ (resp. $\rho_{a}$ ), we have $\mathscr{M}(A)=\oplus \mathscr{M}(A)_{n}$ where $\mathscr{M}(A)_{n}=\operatorname{span}_{k}\left\{\lambda_{a}, \rho_{b} \mid a, b \in A_{n}\right\}$. Alternatively, $\operatorname{End}_{k}(A)$ is a graded $k$-algebra whose subspace of homogeneous elements of degree $n$ is $\oplus_{j=-\infty}^{\infty} \operatorname{Hom}_{k}\left(A_{j}, A_{j+n}\right)$. Clearly, $\mathscr{M}(A)$ is a graded subalgebra of End $_{k} A$ and if $A$ is positively graded, $\mathscr{M}(A)$, with the induced grading, is again positively graded.

The following lemma appears in [3, Chapter 5 and $\mathbf{8}$, Chapter 6].

(3.5) Lemma. Let $B$ be an associative unital graded $k$-algebra of finite type. Suppose further that $B$ is positively graded by finite dimensional subspaces. Let $M=\bigoplus_{i=0}^{\infty} M$ be a finitely generated graded B-module graded by finite dimensional subspaces. Set

$$
g(n)=\sum_{i=0}^{n} \operatorname{dim}_{k} M_{i}
$$

Then $w\left({ }_{B} M\right)=w(g)$.

(3.6) Corollary. Let $A$ be a finite type, positively graded, $k$-algebra that is not necessarily associative. If $A=\bigoplus_{i=0}^{\infty} A_{i}$ and $g(n)=\sum_{i=0}^{n} \operatorname{dim}_{k} A_{i}$, then $w(A) \leqslant w(g)$ with equality if $\mathscr{M}(A)$ is of finite type. 
Proof. Lemma (3.4) yields $w\left(\mathscr{M}(A)^{A}\right)=w(A)$. If $U \subset \mathscr{M}(A)$ and $V \subset A$ are finite dimensional then $U \subseteq \sum_{i=0}^{n} \mathscr{M}(A)_{i}$ and $V \subseteq \sum_{j=0}^{m} A_{m}$ for some $m, n$. Clearly, $U^{i} V \subset$ $\sum_{j=0}^{i n+m} A_{j}$ for all $i$, from which $w(\mathcal{M}(A) A) \leqslant w(g)$ follows. Lemma (3.5) gives equality for $\mathscr{M}(A)$ finite type.

As an example, consider $G_{d}$, the generic algebra for $d$-dimensional $k$-algebras. $G_{d}$ is free as a $k\left[t_{i j} \mid 1 \leqslant i, j, k \leqslant d\right]=k[T]$ module with basis $e^{1}, \ldots, e^{d}$ satisfying $e^{i} e^{j}=\sum_{k=1}^{d} t_{i j}^{k} e^{k}$. We compute $\operatorname{Dim} G_{d}$.

As a $k[T]$-submodule of $\operatorname{End}_{k[T]}\left(G_{d}\right), \mathscr{M}\left(G_{d}\right)$ is finitely generated and torsion free. The following standard lemmas apply.

(3.7) Lemma [7, Proposition 5.5]. If $B$ is a subalgebra of the associative algebra $A$ and $A$ is finitely generated as a left or right $B$-module, then $\operatorname{Dim} B=\operatorname{Dim} A$.

(3.8) Lemma. Dim $k[X]=|X|$ for $X$ a finite set.

Thus $d^{3}=\operatorname{Dim} k[T]=\operatorname{Dim} \mathscr{M}\left(G_{d}\right)$. Furthermore, $\bigcap_{i=1}^{d} \operatorname{Ann}_{\mathscr{M}_{\left(G_{d}\right)}}\left(e^{i}\right)=(0)$, so by (3.3) and (3.4) $\operatorname{Dim} G_{d}=d^{3}$.

IV. The algebra of polynomial functions on a nonassociative algebra. Given an arbitrary algebra $A$ over a commutative, associative unital ring $R$, the algebra of formal polynomials in a set $X$ is the solution to the following universal problem: for all $R$-algebras $B$ with an $R$-algebra homomorphism $\psi: A \rightarrow B$ and a set map $\phi$ : $X \rightarrow B, A\langle X\rangle_{\mathrm{fl}}$ is the unique (up to isomorphy) algebra with algebra homomorphisms $\psi_{0}: A \rightarrow A\langle X\rangle_{\mathrm{fl}}, \psi_{1}: A\langle X\rangle_{\mathrm{fl}} \rightarrow B$ and set map $\Psi_{0} X \rightarrow A\langle X\rangle_{\mathrm{fl}}$ making the following diagram commute:

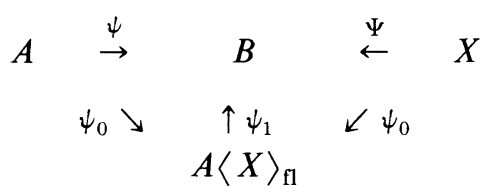

Full details can be found in [10], but it should be noted that $A\langle X\rangle_{\mathrm{fl}}$ can be realized as $R\{X\} \amalg A$, and thus its elements are represented as $R$ linear combinations of formal words whose letters come from $A \cup X$, and are bracketted in a meaningful way.

Evaluation of a formal polynomial amounts to a set map $\varepsilon: X \rightarrow A$ which, together with id: $A \rightarrow A$, induces an algebra homomorphism $\varepsilon^{*}: A\langle X\rangle_{\mathrm{fl}} \rightarrow A$. A formal polynomial $P$ is called inessential if $\varepsilon^{*}(P)=0$ for all $\varepsilon: X \rightarrow A$. The ideal $\bigcap_{\varepsilon: X \rightarrow A} \operatorname{ker} \varepsilon^{*}$ is denoted $\operatorname{In}(A, X)$ and the quotient $A\langle X\rangle_{\mathrm{fl}} / \operatorname{In}(A, X)$ is the algebra of polynomial functions on $A$ in the set $X$, denoted $A\langle X\rangle$. When $X=\{x\}$ we write $A\langle x\rangle$ in place of $A\langle\{x\}\rangle$.

In case $R$ contains an infinite field, a Vandermonde determinant argument [10 or 3] shows that $\operatorname{In}(A, X)$ is a homogeneous ideal with respect to the grading by degree in $X$, so that $A\langle X\rangle$ inherits this grading.

Certain formal properties of $A\langle X\rangle$ needed in the subsequent development are collected in the following proposition. Their proofs can be found in [11 or 3]. 
(4.1) Proposition. (1) If $X$ and $Y$ are disjoint sets, then $A\langle X \cup Y\rangle \cong(A\langle X\rangle)\langle Y\rangle$. (2) If $\left\{A_{i} \mid i \in I\right\}$ is a family of $R$-algebras and $X$ a set, then there is an injective homomorphism $\left(\Pi A_{i}\right)\langle X\rangle \rightarrow \prod A_{i}\left\langle X_{i}\right\rangle$ which is surjective if $I$ is finite and $\mathscr{M}^{*}\left(A_{i}\right)$ $=\mathscr{M}\left(A_{i}\right)$ for each $i$.

(3) If $S$ is a commutative associative unital $R$-algebra, there is a surjective $S$-algebra homomorphism $S \otimes_{R} A\langle X\rangle \rightarrow\left(S \otimes_{R} A\right)\langle X\rangle$ which is injective if $S$ is a free $R$-module.

With the identifications $\lambda_{a} \mapsto a x$ and $\rho_{a} \mapsto x a$, it is apparent that $\mathscr{M}(A)$ can be identified with the submodule $A\langle x\rangle^{(1)}$ of $A\langle x\rangle$ consisting of polynomial functions of degree one in the single indeterminate $x$.

In case $A$ is free as an $R$-module, Röhrl [10] proved the following embedding theorem which allows for a convenient representation of polynomials in $A\langle X\rangle$ and brings the study of their zeros into the realm of classical algebraic geometry $[\mathbf{1 0}, \mathbf{4}]$.

(4.2) THEOREM. If $A$ is free as an $R$-module with basis $e^{1}, \ldots, e^{d}$, then for any set $X$ the algebra homomorphism $A \rightarrow k\left[X_{1}, \ldots, X_{d}\right] \otimes_{R} A$ given by $a \mapsto 1 \otimes a$ and set map $X \rightarrow k\left[X_{1}, \ldots, X_{d}\right]$ given by $x \mapsto \sum_{i=1}^{d} x_{i} \otimes e^{i}$ (where $X_{i}$ is a copy of $X$ and $x_{i} \in X_{i}$ the copy of $x \in X$ ) induce an injective $R$-algebra homomorphism $\chi: A\langle X\rangle \rightarrow$ $R\left[X_{1}, \ldots, X_{d}\right] \otimes_{R} A$.

An immediate consequence of (2.3) and (4.2) is that for $A$ finite dimensional over a field, the growth of $A(x)$ is polynomial bounded (i.e. $w(A\langle X\rangle) \leqslant w(P)$ for some polynomial $P$ ). Indeed, if $\operatorname{dim}_{k} A=d, \operatorname{Dim} A\langle x\rangle \leqslant \operatorname{Dim}_{k}\left[x_{1}, \ldots, x_{d}\right] \otimes A=d$.

It should be noted that if $A$ is commutative, associative, unital, then $A\langle X\rangle \cong A[X]$ the ordinary polynomial ring over $A$. Since G-K dimension agrees with Krull dimension [1] in this case, and $A\langle X\rangle$ is of finite type if $X$ is finite, polynomial growth is clear.

Ideals in $A$ and $A\langle X\rangle$ are related by the assignments,

$$
\begin{aligned}
& I \Delta A \rightarrow\left\{P \in A(X) \mid \varepsilon^{*}(P) \in I \forall \varepsilon: X \rightarrow A\right\}=\mathscr{I} \Delta A\langle X\rangle, \\
& \mathscr{I} \Delta A\langle X\rangle \rightarrow \mathscr{I} \cdot A=\bigcup_{\varepsilon: X \rightarrow A}\left\{\varepsilon^{*}(p) \mid p \in \mathscr{I}\right\} \Delta A .
\end{aligned}
$$

If $A$ is finitely generated free as $R$ module, the evaluation of a polynomial can be carried out in a convenient manner via (4.2). Indeed, for any set map $\psi: X \rightarrow A$ we have the evaluation homomorphism $\psi^{*}: A\langle X\rangle_{\mathrm{fl}} \rightarrow A$ and the evaluation homomorphism $A\langle X\rangle \rightarrow A$ also denoted $\psi^{*}$. With a fixed basis for $A$, say $e^{1}, \ldots, e^{d}$, we can write $\psi(x)=\sum r_{i} e^{i}$ where $r_{i} \in R$, and for $P=P\left(x_{1}, \ldots, x_{n}\right) \in A(X)$ we have

$$
\chi(P)=\sum P_{i}\left(x_{11}, \ldots, x_{1 d}, x_{21}, \ldots, x_{2 d}, \ldots, x_{n d}\right) \otimes e^{i}
$$

in $R\left[X_{1}, \ldots, X_{d}\right] \otimes A$. If we set $\Psi: X_{1} \cup \cdots \cup X_{d} \rightarrow R$ to be the set map $x_{i j} \mapsto r_{i j}$ where $\psi\left(x_{j}\right)=\sum r_{i j} e^{j}$, and $\Psi^{*}: R\left[X_{1}, \ldots, X_{d}\right] \rightarrow R$ the induced algebra homomorphism, then we have a commutative diagram:

$$
\begin{array}{ccc}
A\langle X\rangle & \stackrel{\chi}{\rightarrow} & R\left[X_{1}, \ldots, X_{d}\right] \otimes_{R} A \\
\psi^{*} \downarrow & & \downarrow \Psi^{*} \otimes \mathrm{id}_{A} \\
A & \stackrel{\cong}{\rightarrow} & R \otimes_{R} A
\end{array}
$$


Thus

$$
\psi^{*}(P)=\sum_{i=1}^{d} P_{i}\left(r_{11}, \ldots, r_{1 d}, \ldots, r_{2 d}, \ldots, r_{n 1}, \ldots, r_{n d}\right) e^{i} .
$$

As a consequence we have the

(4.3) LeMma. If $A$ is finite dimensional over a field and $I$ is an ideal of $A$, then $A\langle X\rangle / \mathscr{I} \cong(A / I)\langle X\rangle$.

Proof. The homomorphism $A \rightarrow A / I \rightarrow(A / I)\langle X\rangle$ and set map $X \rightarrow$ $(A / I)\langle X\rangle$ into the degree 0 and degree one subspaces of $(A / I)\langle X\rangle$ induces a surjective homomorphism $\tilde{\pi}: A\langle X\rangle_{\mathrm{fl}} \rightarrow(A / I)\langle X\rangle$ by the definition of $A\langle X\rangle_{\mathrm{fl}}$ and the fact that $(A / I)\langle X\rangle$ is generated as an algebra by the images of $A / I$ and $X$. Clearly, $\operatorname{In}(A, X) \subset$ ker $\tilde{\pi}$ and $\mathscr{I}$ is contained in the kernel of the induced surjection $\pi: A\langle X\rangle \rightarrow(A / I)\langle X\rangle$, inducing a surjection $A\langle X\rangle / \mathscr{I} \rightarrow(A / I)\langle X\rangle$.

To see that $\mathscr{I}=\operatorname{ker} \pi$, choose a basis $e^{1}, \ldots, e^{d}$ for $A$ with $e^{1}, \ldots, e^{l}$ a basis for $I$. Commutativity of the following diagram for all $\varepsilon: X \rightarrow A$ yields $\operatorname{ker} \pi \subset \mathscr{I}$.

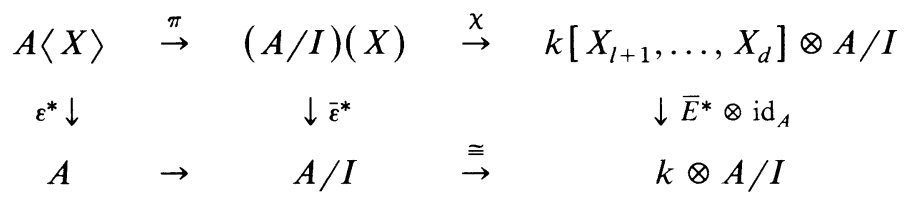

(Here $\bar{\varepsilon}^{*}$ is the homomorphism $(A / I)(X) \rightarrow A / I$ induced from the set map $\bar{\varepsilon}$ obtained from $\varepsilon$ by composing with the canonical map $A \rightarrow A / I$.)

The prime radical of an associative ring consists of all strongly nilpotent elements of that ring [2, vol. II, p. 255]. The corresponding idea for a nonassociative algebra leads to the $\Gamma$ radical [11 or 3]. For $a \in A$, let $\varepsilon_{a}^{*}$ denote the evaluation homomorphism $\varepsilon^{*}: A\langle x\rangle \rightarrow A$ induced by $\varepsilon_{a}: x \mapsto a$. Denote by $A\langle x\rangle^{\geqslant n}$ the ideal in $A\langle x\rangle$ consisting of all polynomials of degree $\geqslant n$, and by $\Gamma^{n}(a)$ the ideal $\varepsilon_{a}^{*}\left(A\langle x\rangle^{\geqslant n}\right)$ in $A$. The readily verified inclusions $\Gamma^{n}(a b) \subset \Gamma^{n}(a) \cap \Gamma^{n}(b)$ and $\Gamma^{2 n}(a+b) \subset$ $\Gamma^{n}(a)+\Gamma^{n}(b)$ allow for the following

(4.4) Definition. The $\Gamma$ radical of $A$, denoted $\gamma(A)$, is the ideal $\left\{a \in A \mid \Gamma^{n}(a)=\right.$ (0) for some $n$.

(4.5) Lemma. Let $A$ be a finite dimensional algebra over a field. Then $\gamma(A\langle X\rangle)=$ $\operatorname{ker}(A\langle X\rangle \rightarrow(A / \gamma(A))\langle X\rangle)$.

Proof. Let $P \in \gamma(A\langle X\rangle)$ and $\varepsilon: X \rightarrow A$. Since $\varepsilon^{*}\left(\Gamma^{n}(P)\right)=\Gamma^{n}\left(\varepsilon^{*}(P)\right)$, we obtain $\varepsilon^{*}(P) \in \gamma(A)$; hence, by (4.3), $\gamma(A\langle X\rangle) \subset \operatorname{ker}(A\langle X\rangle \rightarrow(A / \gamma(A))\langle X\rangle$.

Conversely, suppose $\varepsilon^{*}(P) \in \gamma(A)$ for all $\varepsilon$ : $X \rightarrow A$. Again $\varepsilon^{*}\left(\Gamma^{n}(P)\right)=$ $\Gamma^{n}\left(\varepsilon^{*}(P)\right)$ shows $\varepsilon^{*}(P) \in \gamma(A)$.

In case $A$ is associative, and of arbitrary dimension, $\gamma(A)$ is equal to the prime radical of $A$. If, in addition, $A$ is right or left artinian, the $\Gamma$ radical and the Jacobson radical coincide [2, vol. I, p. 398].

Certain improved bounds on $\operatorname{Dim} A\langle x\rangle$ can be derived from the following representation of a polynomial. Given a monomial $M \in A\langle x\rangle$ of degree $n$, label the occurrences of $x$ with $x_{1}, \ldots, x_{n}$, obtaining a multilinear homogeneous element 
$\tilde{M} \in A\left\langle x_{1}, \ldots, x_{n}\right\rangle$ of degree $n$. Under the surjection $A\left\langle x_{1}, \ldots, x_{n}\right\rangle \rightarrow A\langle x\rangle$ induced by $x_{1} \mapsto x$ for each $i$ and $a \mapsto a$ for each $a \in A$, it is clear that $\tilde{M} \rightarrow M$. Furthermore,

$$
\chi(\tilde{M})=\sum_{i \leqslant i_{1}, \ldots, i_{n} \leqslant d} x_{1_{i_{1}}} x_{2_{i_{2}}} \cdots x_{n_{i_{n}}} \otimes \tilde{M}\left(e^{i_{1}}, \ldots, e^{i_{n}}\right)
$$

and

$$
\chi(M)=\sum_{1 \leqslant i_{1}, \ldots, i_{d} \leqslant n} x_{i_{1}} \cdots x_{i_{n}} \otimes \tilde{M}\left(e^{i_{1}}, \ldots, e^{i_{n}}\right) .
$$

By taking sums of such monomials we can express $\chi(f)$ for any homogeneous $f \in A\langle x\rangle^{n}$ as

$$
\chi(f)=\sum_{1 \leqslant i_{1} \leqslant i_{2} \leqslant \ldots \leqslant d} x_{i_{1}}, \ldots, x_{i_{n}} \otimes \tilde{f}\left(e^{i_{1}}, \ldots, e^{i_{n}}\right)
$$

where $\tilde{f} \in A\left\langle x_{1}, \ldots, x_{n}\right\rangle$ is multilinear homogeneous of degree $n$.

(4.7) Definition. (1) An algebra $A$ is said to be a subdirect product of a family $\left\{A_{i}: i \in I\right\}$ of algebras provided $A$ is isomorphic to a subalgebra of the product $\prod_{i \in I} A_{i}$ and the composition $A \rightarrow \prod_{i \in I} A_{i} \stackrel{p_{j}}{\rightarrow} A_{j}$ is surjective for all $j \in I$, where $p_{j}$ is the jth projection.

(2) $A$ is said to be subdirectly irreducible provided that in every representation of $A$ as a subdirect product $\varepsilon: A \rightarrow \prod_{i \in I} A_{i}, p_{j} \circ \varepsilon$ is an isomorphism for at least one $j$. Otherwise $A$ is said to be subdirectly reducible.

It should be noted that subdirect reducibility is equivalent to $\bigcap_{0 \neq I \Delta A} I=(0)$ since in this case, the homomorphism $\prod_{0 \neq I \triangle A} q_{I}: A \rightarrow \prod_{0 \neq I \Delta A} A / I$ is injective, where $q_{I}: A \rightarrow A / I$ is the canonical map. Thus subdirect irreducibility is equivalent to the existence of a unique nonzero minimal ideal.

(4.8) Proposition. Let $A$ be a finite dimensional k-algebra. Then

(1) $\operatorname{Dim} A\langle x\rangle \leqslant \operatorname{dim}_{k} A-\operatorname{dim}_{k} \gamma(A)$.

(2) If $A$ is subdirectly reducible, $\operatorname{Dim} A\langle x\rangle \leqslant \max _{0 \neq I \Delta A} \operatorname{dim}_{k} A / I$.

Proof. (1) Let $e^{1}, \ldots, e^{d}$ be a $k$ basis for $A$ with $e^{1}, \ldots, e^{g}$ a basis for $\gamma(A)$. Let $q$ be the nilpotency degree of $\gamma(A)$, and $M \in A\langle x\rangle^{q}$ a nonzero monomial. From (4.6)

$$
\chi(M)=\sum_{1 \leqslant i_{1}, \ldots, i_{2} \leqslant d} x_{i_{1}} \cdots x_{i_{n}} \otimes \tilde{M}\left(e^{i_{1}}, \ldots, e^{i_{q}}\right),
$$

and from the definition of $q$, it is clear that the nonzero summands in $\chi(M)$ are of degree at most $q-1$ in $x_{1}, \ldots, x_{g}$. In particular,

$$
\operatorname{im} \chi \subset \sum_{j_{1}+\cdots+j_{g} \leqslant q-1} x_{1}^{j_{1}} \cdots x_{g}^{j_{g}} k\left[x_{g+1}, \ldots, x_{d}\right] \otimes A .
$$

Since

$$
\sum_{j_{1}+\cdots+j_{g} \leqslant q-1} x_{1}^{j_{1}} \cdots x_{g}^{j_{g}} k\left[x_{g+1}, \ldots, x_{d}\right] \otimes A
$$

is a finitely generated $k\left[x_{g+1}, \ldots, x_{d}\right]$ module, we obtain $\operatorname{Dim} A\langle x\rangle \leqslant$ $\operatorname{Dim} k\left[x_{g+1}, \ldots, x_{d}\right]=d-g$. 
(2) Since $A$ is finite dimensional and subdirectly reducible, there is a finite collection of nonzero ideals $I_{1}, \ldots, I_{n}$ satisfying $I_{1} \cap I_{2} \cap \cdots \cap I_{n}=(0)$. Thus $q \equiv \Pi_{i=1}^{n} q_{i}: A \rightarrow A / I_{1} \Pi \cdots \Pi A / I_{n}$ expresses $A$ as a product of algebras of strictly smaller dimension. The universal property defining $A\langle X\rangle_{\mathrm{fl}}$ applied to $q$ and the map $x \mapsto x$ gives rise to a monomorphism $A\langle X\rangle_{\mathrm{f} 1} \rightarrow \Pi_{j}\left(A / I_{j}\right)\langle X\rangle_{\mathrm{fl}}$. One checks that the image of $\operatorname{In}(A, X)$ lies inside $\operatorname{In}\left(\Pi_{j} A / I_{j}, X\right)$, inducing a homomorphism $q\langle X\rangle: A\langle X\rangle \rightarrow \prod_{j}\left(A / I_{j}\right)\langle X\rangle$. If $P \in \operatorname{ker} q(X)$, then $\varepsilon^{*} \cdot q(X)(P)=0$ for all $\varepsilon$ : $X \rightarrow \prod_{j} A / I_{j}$ from which $\varepsilon^{*}(P) \subset \cap_{j} I_{j}=(0)$ follows. Thus $q\langle X\rangle$ is a monomorphism, and $q$ induces a subdirect decomposition $q\langle X\rangle$ of $A\langle X\rangle$.

The result now follows from (2.4) and the remarks following (4.2).

(4.9) CoRollary. An indeterminate $x_{i}$ occurs to only finitely many powers among the coordinate polynomials in the image of $\chi$ iff $e^{i} \in \gamma(A)$.

Proof. $(\Leftarrow)$ is contained in the proof of Lemma (4.8).

$(\Rightarrow)$ if $e^{\alpha} \notin \gamma(A)$, then for each $l>0, \Gamma^{l}\left(e^{\alpha}\right) \neq(0)$. In particular, there is a monomial $M \in A(x)^{l}$ whose evaluation at $e^{\alpha}$ is nonzero:

$$
\chi(M)=\sum_{1 \leqslant i_{1}, \ldots, i_{l} \leqslant d} x_{i_{1}} \cdots x_{i_{l}} \otimes \tilde{M}\left(e^{i_{1}}, \ldots, e^{i_{l}}\right) .
$$

Evidently, the $x_{\alpha}^{l}$ term is nonzero.

(4.10) Theorem. Let $A$ be a finite dimensional $k$-algebra. Then the following are equivalent:

(1) $\operatorname{Dim} A\langle x\rangle=\operatorname{dim}_{k} A$.

(2) $A$ is subdirectly irreducible with unique nonzero minimal ideal I satisfying $I^{2} \neq(0)$ and $L \otimes_{k} A$ has this property for all field extensions $L$ of $k$.

(3) $A$ is subdirectly irreducible with minimal ideal $I$ satisfying $I^{2} \neq(0)$ and $\mathscr{M}(A) /$ Ann $I \cong$ End $_{k} I$.

Proof. (1) $\Rightarrow(2)$ From Proposition (4.8) we have $A$ subdirectly irreducible. If $I^{2}=(0)$ then $I \subset \gamma(A)$ since $\Gamma^{2}(a) \subset I^{2}$ for any $a \in I$. But $\gamma(A)=(0)$, again by (4.8). Since $L \otimes A\langle x\rangle \cong(L \otimes A)\langle x\rangle$, (4.1), and $\operatorname{Dim} L \otimes A\langle x\rangle=\operatorname{Dim} A\langle x\rangle$ we have (2).

(2) $\Rightarrow(3) \mathscr{M}(A) /$ Ann $I$ is a simple associative $k$-algebra, by the minimality of $I$ as an $\mathscr{M}(A)$ module. If $\mathscr{M}(A) /$ Ann $I$ is not $k$ central, then $\bar{k} \otimes \mathscr{M}(A) /$ Ann $I$ is isomorphic to a product of $\bar{k}$-algebras and $\bar{k} \otimes I$ is a direct sum of nonzero ideals of $\bar{k} \otimes A$, contradicting (2).

Suppose then that $\mathscr{M}(A) /$ Ann $I \cong$ End $_{D} I$ for some $k$ central division algebra $D$ with $n^{2}=\operatorname{dim}_{k} D$. Let $r=\operatorname{dim}_{D} I$ and note that $\bar{k} \otimes \mathscr{M}(A) /$ Ann $I \cong M_{n r}(\bar{k})$. Thus $\bar{k} \otimes I$ is isomorphic to a direct sum of simple $M_{n r}(\bar{k})$ submodules of $\bar{k} \otimes A$ each of dimension $n r$ over $\bar{k}$. But $\mathscr{M}(\bar{k} \otimes A) / \operatorname{Ann}(\bar{k} \otimes I) \cong \mathscr{M}_{n r}(\bar{k})$ so that $\bar{k} \otimes I$ is isomorphic to a direct sum of ideals, again contradicting (2).

$(3) \Rightarrow(2) \quad \bar{k} \otimes \mathscr{M}(A) / \operatorname{Ann} I \cong \bar{k} \otimes \mathscr{M}(A) / \bar{k} \otimes$ Ann $I \cong \operatorname{End}_{\bar{k}}(\bar{k} \otimes I)$. Moreover, by $(3.1)^{\prime}, \bar{k} \otimes \mathscr{M}(A) \cong \mathscr{M}(\bar{k} \otimes A)$, and the image of $\bar{k} \otimes$ Ann $I$ is contained in $\operatorname{Ann}(\bar{k} \otimes I)$. But simplicity of $\operatorname{End}_{\bar{k}}(\bar{k} \otimes I)$ forces $\bar{k} \otimes$ Ann $I \cong \operatorname{Ann} \bar{k} \otimes I$, so 
that $\bar{k} \otimes \mathscr{M}(A) / \operatorname{Ann} I \cong \mathscr{M}(\bar{k} \otimes A) / \operatorname{Ann}(\bar{k} \otimes I) \cong \operatorname{End}_{\bar{k}}(\bar{k} \otimes I)$. Thus $\mathscr{M}(\bar{k} \otimes A)$ acts densely on $\bar{k} \otimes I$, proving that $\bar{k} \otimes I$ is the unique minimal ideal of $\bar{k} \otimes A$.

(2) $\Rightarrow$ (1) Without loss, we assume $k$ is algebraically closed and $A$ has a unique minimal ideal $I$ with $I^{2} \neq(0)$. Choose a basis $e^{1}, \ldots, e^{d}$ for $A$ compatible with a composition series of ideals (left $\mathscr{M}(A)$ submodules) of $A:(0)=I_{0} \subset I=I_{1} \subset \cdots$ $\subset I_{n}=A$ (i.e., $e^{1}, \ldots, e^{l_{1}}$ is a basis for $I_{1}, e^{1}, \ldots, e^{l_{1}}, e^{l_{1}+1}, \ldots, e^{l_{1}+l_{2}}$ is a basis for $I_{2}$, etc). Relative to this basis, the matrices in $\mathscr{M}(A)$ have block upper triangular form.

Let $\mathscr{J}$ denote the radical of $\mathscr{M}(A)$. Since $k$ is algebraically closed, the Wedderburn principal theorem [6, p. 116] asserts that $\mathscr{M}(A) \cong\left(\prod_{i=1}^{n} R_{i}\right) \oplus \mathscr{J}$, where the $R_{i}$ are simple $k$-algebras. Relative to the above basis for $A$, the matrices corresponding to $R$ can be taken to be the upper left block of $\operatorname{dim} I \times \operatorname{dim} I$ matrices. Let $\tau_{11}$ be the idempotent in $\mathscr{M}(A)$ defined by $\tau_{11}: e^{j} \mapsto \delta_{1 j} e^{1}$ where $\delta_{1 j}$ is the Kronecker $\delta$.

We show $\operatorname{Dim} A\langle x\rangle=\operatorname{dim} A=d$ by showing that the ideal $\mathscr{I}=\{p \in$ $A\langle x\rangle \mid \varepsilon^{*}(P) \in I$ for all $\left.\varepsilon:\{x\} \rightarrow A\right\}$ is isomorphic to $k\left[x_{1}, \ldots, x_{d}\right] \otimes I$. The proof is by induction on degree, with the degree 0 case trivial.

To prove that for any linear homogeneous $f \in k\left[x_{1}, \ldots, x_{d}\right], f \otimes a \in \mathscr{I}$ for all $a \in I$, it suffices to prove that the vector space dimension of the right ideal $\tau_{11} \mathscr{M}(A)$ is exactly $d$. Suppose $\operatorname{dim}_{k} \tau_{11} \mathscr{M}(A)=l<d$, and $\sigma_{1}, \ldots, \sigma_{l}$ is a basis. Consider the linear system

$$
\boldsymbol{\sigma}_{i}\left[\begin{array}{c}
y_{1} \\
\vdots \\
y_{d}
\end{array}\right]=\left[\begin{array}{c}
0 \\
0
\end{array}\right], \quad 1 \leqslant i \leqslant l,
$$

where $\sigma_{i}$ are matrices with nonzero entries only in the first row. These are $l$ linear equations in $d$ unknowns for which a nontrivial solution $\left(\alpha_{1}, \ldots, \alpha_{d}\right)$ exists. Let $a=\sum \alpha_{i} e^{1}$. By minimality of $I, I \subset \mathscr{M}(A) a$, but $e^{1} \in \tau_{11} I \subset \tau_{11} \mathscr{M}(A) a=(0)$, a contradiction.

Now suppose that for all monomials $M$ of degree $n$ in $x_{1}, \ldots, x_{d}, M \otimes a \in \mathscr{I}$ for all $a \in I$. Let $x_{1}^{\alpha_{1}} \cdots x_{d}^{\alpha_{d}}$ have degree $n+1$, and assume $\alpha_{i} \neq 0$. Then for each $a \in I, x_{1}^{\alpha_{1}} \cdots x_{i}^{\alpha_{i}-1} \cdots x_{d}^{\alpha_{a}} \otimes a \in \mathscr{I}$. Choose $a, b \in I$ with $a b \neq 0$. Then

$$
\left(x_{1}^{\alpha_{1}} \cdots x_{i}^{\alpha_{i}-1} \cdots x_{d}^{\alpha_{d}} \otimes a\right)\left(x_{i} \otimes_{1} b\right)=x_{i}^{\alpha_{1}} \cdots x_{d}^{\alpha_{1}} \cdots x^{\alpha_{d}} \otimes a b
$$

is nonzero and in the image of $\chi$. Since $\mathscr{M}(A)$ acts densely on $I$ we have $\mathscr{I} \cong k\left[x_{1}, \ldots, x_{d}\right] \otimes I$.

An important special case of this theorem is the case of $A$ strictly simple [10] (i.e. $A$ is simple and remains so under all base extensions $L \otimes A$ to field extensions $L$ of $k$ ). The monomorphism $\chi$ is an isomorphism precisely when $A$ is strictly simple.

If $A$ is simple, but not strictly simple, then $A$ is strictly simple viewed as an algebra over its centroid $Z$, a proper field extensions of $k$. In this case, $A\langle x\rangle \cong$ $Z\left[x_{1}, \ldots, x_{d}^{\prime}\right] \otimes_{Z} A$ where $d=d^{\prime} \cdot[Z: k]$. By Lemma (2.5), $\operatorname{Dim} A\langle x\rangle=d^{\prime}=$ $(\operatorname{dim} A) /[Z: k]$. 
V. Integrality theorem for associative algebras. Let $A$ be an associative unital algebra finite dimensional over the field $k$. Since $(\bar{k} \otimes A)\langle x\rangle \cong \bar{k} \otimes_{k} A\langle x\rangle$ and $\operatorname{Dim}(\bar{k} \otimes A)\langle x\rangle=\operatorname{Dim} A\langle x\rangle$, we will assume that $k$ is algebraically closed. Denote by $J$ the Jacobson radical of $A$. Then $A / J \cong \Pi R_{i}$ where the $R_{i}$ are simple $k$-algebras. The Wedderburn principal theorem asserts that $A$ has a separable subalgebra $S$ isomorphic to $\prod_{i} R_{i}$ so that $A \cong S \oplus J$. Moreover, one easily shows [3] that $1_{s}=1_{A}$. We identify each $R_{i}$ with its image in $S$.

The Wedderburn decomposition of $A$ extends to $A\langle X\rangle$ as follows: Choose a basis for $A$ of the form

$$
\left\{e_{1}^{1}, \ldots, e_{1}^{d_{1}}\right\} \cup\left\{e_{2}^{1}, \ldots, e_{2}^{d_{2}}\right\} \cup \cdots \cup\left\{e_{n}^{1}, \ldots, e_{n}^{d_{n}}\right\} \cup\left\{f^{1}, \ldots, f^{s}\right\}
$$

where $\left\{e_{j}^{i}\right\}$ constitutes a basis for $R_{j}$ and $\left\{f^{i}\right\}$ constitutes a basis for $J$. Relative to this basis,

$$
\chi: A\langle X\rangle \rightarrow k\left[X_{1}^{(1)}, \ldots, X_{d_{1}}^{(1)}, \ldots, X_{1}^{(n)}, \ldots, X_{d_{n}}^{(n)}, Y_{1}, \ldots, Y_{s}\right] \otimes A
$$

and

$$
\begin{gathered}
k\left[X_{1}^{(1)}, \ldots, X_{d_{1}}^{(1)}, \ldots, X_{1}^{(n)}, \ldots, X_{d_{n}}^{(n)}, Y_{1}, \ldots, Y_{s}\right] \otimes A \\
\quad \cong k\left[X_{1}^{(1)}, \ldots, X_{d_{n}}^{(n)}, Y_{1}, \ldots, Y_{s}\right] \otimes\left(\prod R_{i} \oplus J\right) .
\end{gathered}
$$

By (4.6), for any monomial term $M$ of positive degree in $Y_{1}, \ldots, Y_{s}$ and any evaluation $\varepsilon:\left(\cup_{i, j} X_{i}^{(j)}\right) \cup\left(\cup_{k} Y_{k}\right) \rightarrow A, \varepsilon^{*}(M) \in J$. Thus, the image of $\chi$ lies in $\left(\prod_{i=1}^{n} k\left[X_{1}^{1}, \ldots, X_{d_{n}}^{(n)}\right] \otimes R_{i}\right) \oplus \mathscr{J}^{\prime}$ where $\mathscr{J}^{\prime}=k\left[X_{1}^{(1)}, \ldots, X_{d_{n}}^{(n)}, Y_{1}, \ldots, Y_{s}\right] \otimes J$ is the Jacobson radical of $k\left[X_{1}^{(1)}, \ldots, X_{d_{n}}^{(n)}, Y_{1}, \ldots, Y_{s}\right] \otimes A$. Indeed, if $\mathscr{J}$ denotes the Jacobson radical of $A\langle X\rangle$,

$$
\begin{aligned}
A\langle X\rangle / \mathscr{J} & \cong(A / J)\langle X\rangle \cong\left(\prod_{i=1}^{n} R_{i}\right)(X) \cong \prod_{i=1}^{n} R_{i}\left(X_{i}\right) \\
& \cong \prod_{i=1}^{n} k\left[X_{1}^{(i)}, \ldots, X_{d_{i}}^{(i)}\right] \otimes R_{i} .
\end{aligned}
$$

We thus obtain $A\langle X\rangle \cong \prod_{i=1}^{n} k\left[X_{1}^{(i)}, \ldots, X_{d_{1}}^{(i)}\right] \otimes R_{i} \oplus \mathscr{J} \quad$ where $\prod_{i=1}^{n} k\left[X_{1}^{(i)}, \ldots, X_{d_{1}}^{(i)}\right] \otimes R_{i}$ is a separable subalgebra of $A\langle X\rangle$.

For each sequence $\sigma=\left(i_{1}, \ldots, i_{l}\right)$ of distinct integers $1 \leqslant i_{j} \leqslant n$, denote by $V(\sigma)$ the $k$-vector subspace $R_{i_{1}} J R_{i_{2}} J \cdots J R_{i_{1}}$ of $A$. Note that $V(\sigma)=(0)$ if $l$ is greater than or equal to the nilpotency degree of $J$.

Set $d_{i}=\operatorname{dim}_{k} R_{i}$, and for each nonzero $V(\sigma)$, set $s(\sigma)=\sum_{i_{j} \in \sigma} d_{i j}$. Let $g$ denote $\operatorname{Max}\left\{\operatorname{Max}_{\sigma}\left\{s(\sigma), \operatorname{Max}_{i}\left\{d_{i}\right\}\right\}\right\}$.

(5.1) ThEOREM. $\operatorname{Dim} A\langle x\rangle=g$.

Proof. Since $\prod_{i=1}^{n} k\left[x_{1}^{(i)}, \ldots, x_{d_{i}}^{(i)}\right] \otimes R_{i}$ is a subalgebra of $A\langle x\rangle$ of G-K dimension $\operatorname{Max}_{i}\left\{d_{i}\right\}$, we obtain $\operatorname{Dim} A\langle x\rangle \geqslant \operatorname{Max}_{i}\left\{d_{i}\right\}$.

Suppose $V(\sigma) \neq(0)$, and let $a=r_{1} a_{1} \cdots a_{l-1} r_{l}$ be a nonzero element in $V(\sigma)$, where $\sigma=\left(i_{1}, \ldots, i_{l}\right), r_{j} \in R_{i_{j}}$, and $a_{j} \in J$. For each $j \in \sigma$, let $M_{j}$ be monomial in 


$$
\begin{gathered}
k\left[x_{1}^{(j)}, \ldots, x_{d_{j}}^{(i)}\right], M_{r_{j}} \in A\langle x\rangle \text { with } \chi\left(M_{r_{j}}\right)=M_{j} \otimes r_{j} . \\
\begin{aligned}
\chi\left(M_{r_{1}} a_{1} M_{r_{2}} a_{2} \cdots a_{l-1} M_{r_{l}}\right) & =\left(M_{1} \otimes r_{1}\right)\left(1 \otimes a_{1}\right) \cdots\left(1 \otimes a_{l-1}\right)\left(M_{l} \otimes r_{l}\right) \\
& =\prod_{i=1}^{l} M_{i} \otimes a \neq 0 .
\end{aligned}
\end{gathered}
$$

Since any monomial in $k\left[\left\{x_{\alpha}^{\left(i_{j}\right)} \mid i_{j} \in \sigma, 1 \leqslant \alpha \leqslant d_{i_{j}}\right\}\right]$ can arise as $\Pi_{i=1}^{l} M_{i}$, and $\operatorname{Dim} k\left[\left\{x_{\alpha}^{\left(i_{j}\right)} \mid i_{j} \in \sigma, 1 \leqslant \alpha \leqslant d_{i_{j}}\right\}\right]$ is exactly $s(\sigma)$, we obtain $\operatorname{Dim} A\langle x\rangle \geqslant g$.

For any $P \in A\langle x\rangle, \chi(P)=\sum_{i, \alpha} P_{i, \alpha} \otimes e_{(i)}^{\alpha}+\sum_{k} Q_{k} \otimes f^{k}$, where the $Q_{k}$ are of degree bounded by $\eta$, the degree of nilpotency of $J$, in $y_{1}, \ldots, y_{s}$. Furthermore, by the decomposition of $A\langle x\rangle$, for each fixed $i, P_{i, \alpha} \in k\left[x_{1}^{(i)}, \ldots, x_{d_{i}}^{(i)}\right]$. In particular, a monomial term involving $x_{\alpha}^{(i)}$ and $x_{\beta}^{(j)}$ for $i \neq j$ can occur only among the $Q_{k}$, and then only if $R_{i} J R_{j}$ or $R_{j} J R_{i}$ is nonzero, using the orthogonality of the distinct $R_{i}$. In particular, such monomial terms can arise only if $V(\sigma) \neq 0$ for $\sigma$ containing $i$ and $j$. Since there are only finitely many sequences $\sigma$, the collection of coordinate polynomials $P_{i, \alpha}$ and $Q_{k}$ arising in the expansions of the polynomials in $A(x)$ can be identified with a subset of a finite sum of vector spaces:

$$
\sum_{\sigma} \sum_{\alpha_{1}+\cdots+\alpha_{s}<\eta} k\left[\left\{x_{\beta}^{\left(i_{j}\right)} \mid i_{j} \in \sigma, 1 \leqslant \beta \leqslant d_{i_{j}}\right] y_{1}^{\alpha_{1}} \cdots y_{s}^{\alpha_{s}} .\right.
$$

If $t(n)$ denotes the dimension of the vector space $\oplus_{i=1}^{n} A\langle x\rangle^{(i)}$, we have $w(t) \leqslant$ $w\left(k\left[x_{\beta}^{\left(i_{j}\right)} \mid i_{j} \in \sigma, 1 \leqslant \beta \leqslant d_{i_{j}}\right]\right)$ for any $\sigma$ with $s(\sigma)=g$. By Lemma (3.5), with $B=A\langle x\rangle=M$, we obtain $\operatorname{Dim} A\langle x\rangle=w(t) \leqslant g$.

A simple example illustrating the theorem is given by $T_{n, k}\langle x\rangle$ the algebra of polynomial functions in one indeterminate over the $n \times n$ upper triangular matrices with entries in $k$. The Jacobson radical, $J$, consists of all strictly upper triangular matrices, and $T_{n, k} / J \cong \prod_{i=1}^{n} k$. Denoting by $e_{i j}$ the $(i, j)$ th matrix unit, we have $e_{1 n}=e_{11} e_{12} e_{22} e_{23} \cdots e_{n-1 n} e_{n n}$ with each $e_{i i+1} \in J$. Thus $\operatorname{Dim} T_{n, k}\langle x\rangle=n=$ $\operatorname{dim} T_{n, k}-\operatorname{dim} J$.

The algebra $T_{2, k}\langle x\rangle$ has the explicit representation

$$
\left[\begin{array}{cc}
k\left[x_{1}\right] & k\left[x_{1}, x_{2}\right]+x_{3} k\left[x_{1}, x_{2}\right] \\
0 & k\left[x_{2}\right]
\end{array}\right]
$$

from which $\operatorname{Dim} T_{2, k}\langle x\rangle=2$ is clear.

By a result of Lorenz and Small [9], $T_{n, k}\langle x\rangle$ is not both right and left Noetherian if $n>1$, since $\operatorname{Dim} T_{n, k}\langle x\rangle / J=\operatorname{Dim} \prod k_{i}\left[x_{i}\right]=1<\operatorname{Dim} T_{n, k}\langle x\rangle=n$. From the representation above, it is clear that $T_{n, k}\langle x\rangle$ is neither right nor left noetherian.

VI. The integrality theorem for general algebras. As in $\S \mathrm{V}$, the base field is assumed to be algebraically closed, and $A$ is assumed to be a finite dimensional $k$-algebra. The integrality of $\operatorname{Dim} A\langle x\rangle$ is obtained by proving $\operatorname{Dim} \mathscr{M}(A\langle x\rangle)$ to be integral. Since $A\langle x\rangle$ is finite type, (3.2) yields $\operatorname{Dim} A\langle x\rangle=\operatorname{Dim}_{\mathscr{M}(A\langle x\rangle)} A\langle x\rangle$. The following lemma together with this observation shows that integrality of $\mathscr{M}(A\langle x\rangle)$ is sufficient. 
(6.1) Lemma. Let $A$ be a finite dimensional $k$ algebra. Then $\operatorname{Dim} A\langle x\rangle=$ $\operatorname{Dim} \mathscr{M}(A\langle x\rangle)$.

Proof. We have observed that $\operatorname{Dim} A\langle x\rangle=\operatorname{Dim}_{\mathscr{M}(A\langle x\rangle)} A\langle x\rangle$. Let $e^{1}, \ldots, e^{d}$ be a basis for $A$, and consider these elements as lying in the degree 0 subspace of $A\langle x\rangle$. Suppose $\Phi \in \mathscr{M}(A\langle x\rangle)$ annihilates $e^{1}, \ldots, e^{d}$. Let $P \in A\langle x\rangle, \varepsilon:\{x\} \rightarrow A$, and $\varepsilon^{*}(P)=\sum \alpha_{i} e^{i}$

$$
\left(\varepsilon^{*} \cdot \Phi\right)(P)=\varepsilon^{*}\left(\Phi \cdot \varepsilon^{*}(P)\right)=\varepsilon^{*} \Sigma \alpha \Phi\left(e^{i}\right)=0 .
$$

Since $\varepsilon$ and $P$ were arbitrary, $\Phi=0$. By Proposition (3.3), $\operatorname{Dim}_{\mathscr{M}(A\langle x\rangle)} A\langle x\rangle=$ $\operatorname{Dim} \mathscr{M}(A\langle x\rangle)$.

In case $k$ is not algebraically closed, we have

$$
\operatorname{Dim} \mathscr{M}(A\langle x\rangle)=\operatorname{Dim} \bar{k} \otimes_{k} \mathscr{M}(A\langle x\rangle)=\operatorname{Dim} \mathscr{M}(\bar{k} \otimes A\langle x\rangle)
$$

by $(3.1)^{\prime}$ and (4.1), so that indeed there is no loss in assuming $k$ to be algebraically closed.

(6.2) Lemma. The monomorphism $X: A\langle X\rangle \rightarrow k\left[X_{1}, \ldots, X_{d}\right] \otimes A$ induces a monomorphism $\Phi_{X}: \mathscr{M}(A\langle X\rangle) \rightarrow k\left[X_{1}, \ldots, X_{d}\right] \otimes \mathscr{M}(A)$.

Proof. Relative to a prescribed basis $e_{1}^{1}, \ldots, e_{1}^{d}, \chi$ sends an element $P \in A(x)$ to $\sum_{i=1}^{d} P_{i} \otimes e^{i}$. Set

$$
\Phi_{X}\left(\lambda_{P}\right)=\sum_{i=1}^{d} P_{i} \otimes \lambda_{e^{i}} \quad \text { and } \quad \Phi_{X}\left(\rho_{P}\right)=\sum_{i=1}^{d} P_{i} \otimes \rho_{e^{i}} .
$$

To see that the extension to $\mathscr{M}(A\langle X\rangle)$ is well defined, consider the following diagram for $Q \in A\langle X\rangle$ :

$$
\begin{array}{ccc}
\mathscr{M}(A\langle X\rangle) & \stackrel{\varepsilon_{Q}}{\rightarrow} & A\langle X\rangle \\
\Phi_{X} \downarrow & & \downarrow \chi \\
k\left[X_{1}, \ldots, X_{d}\right] \otimes \mathscr{M}(A) & \stackrel{\tilde{\varepsilon}_{Q}}{\rightarrow} & k\left[X_{1}, \ldots, X_{d}\right] \otimes A
\end{array}
$$

where $\varepsilon_{Q}(\tau)=\tau(Q)$ for $\tau \in \mathscr{M}(A\langle X\rangle)$, and $\tilde{\varepsilon}_{Q}\left(\sum s_{i} \otimes \tau_{i}\right)=\sum_{i, j=1}^{d} Q_{i} s_{j} \otimes \tau_{i}\left(e^{j}\right)$. Commutativity is easily verified, from which one deduces that $\Phi_{X}$ is well defined and injective.

Let $J$ denote the Jacobson radical of $\mathscr{M}(A)$, and let $\mathscr{M}(A) \cong\left(\Pi_{i=1}^{n} R_{i}\right) \oplus J$ be a Wedderburn decomposition. With respect to the grading on $\mathscr{M}(A\langle X\rangle)$ by degree in $X, \mathscr{M}(A)$ is isomorphic to the degree zero subspace. Consequently, $\prod_{i=1}^{n} R_{i}$ is isomorphic to a separable subalgebra of $\mathscr{M}(A\langle X\rangle)$. We identify $\Pi R_{i}$ with its image in $\mathscr{M}(A)$ and $\mathscr{M}(A)$ with its image in $\mathscr{M}(A\langle X\rangle)$.

Note that $k\left[X_{1}, \ldots, X_{d}\right] \otimes J$ is a nilpotent homogeneous ideal in $k\left[X_{1}, \ldots, X_{d}\right] \otimes$ $\mathscr{M}(A)$, modulo which $k\left[X_{1}, \ldots, X_{d}\right] \otimes \mathscr{M}(A)$ is semisimple. Thus, $k\left[X_{1}, \ldots, X_{d}\right] \otimes J$ is the Jacobson radical of $k\left[X_{1}, \ldots, X_{d}\right] \otimes \mathscr{M}(A)$. We have a graded $k$-algebra homomorphism:

$$
\mathscr{M}(A\langle X\rangle) \stackrel{\Phi_{X}}{\rightarrow} k\left[X_{1}, \ldots, X_{d}\right] \otimes \mathscr{M}(A) \stackrel{q}{\rightarrow} \prod_{i=1}^{n} k\left[X_{1}, \ldots, X_{d}\right] \otimes R_{i}
$$


where $q$ is the quotient homomorphism. The kernel of $q \circ \Phi_{X}$ is a homogeneous nilpotent ideal in $\mathscr{M}(A\langle X\rangle)$, denoted $\tilde{\mathscr{J}}$. Denote by $\tilde{\Phi}_{X}$ the induced graded monomorphism

$$
\tilde{\Phi}_{X}: \mathscr{M}(A\langle X\rangle) / \tilde{\mathscr{J}} \rightarrow \prod k\left[X_{1}, \ldots, X_{d}\right] \otimes R_{i} .
$$

Let

$$
\pi_{\alpha}: \prod_{i=1}^{n} k\left[X_{1}, \ldots, X_{d}\right] \otimes R_{i} \rightarrow k\left[X_{1}, \ldots, X_{d}\right] \otimes R_{\alpha}
$$

be the $\alpha$ th projection. The image of $\pi_{\alpha} \circ \tilde{\Phi}_{X}$ is a graded subalgebra $S_{X, \alpha} \otimes R_{\alpha}$ of $k\left[X_{1}, \ldots, X_{d}\right] \otimes R_{\alpha}$, and $S_{X, \alpha}$ is a graded subalgebra of $k\left[X_{1}, \ldots, X_{d}\right]$. This follows because $\Phi_{X}, \tilde{\Phi}_{X}$, and $\pi_{\alpha}$ are all graded and the image of $\pi_{\alpha} \circ \tilde{\Phi}_{X}$ contains an isomorphic copy of the central simple algebra $R_{\alpha}$.

Consider the case $X=\{x\}$. To simplify the notation we drop the subscript $\{x\}$ in $\Phi_{\{x\}}, \tilde{\Phi}_{\{x\}}, \tilde{J}_{\{x\}}$, and $S_{\{x\}, \alpha^{*}}$ Note that $\operatorname{Dim} S_{\alpha} \leqslant d$ for each $1 \leqslant \alpha \leqslant n$. For each sequence of distinct integers $\sigma=\left(i_{1}, \ldots, i_{l}\right), 1 \leqslant i_{j} \leqslant n$, indexing the simple factors $\mathscr{M}(A) / J$, let $V(\sigma)$ be the subspace $R_{i_{1}} J R_{i_{2}} J \cdots J R_{i_{l}}$ of $\mathscr{M}(A)$, and let $k[\sigma]$ be the graded subalgebra of $k\left[x_{1}, \ldots, x_{d}\right]$ generated by $\left\{S_{i_{j}} \mid i_{j} \in \sigma\right\}$. Set

$$
g=\operatorname{Max}\left\{\operatorname{Max}_{\sigma}\{\operatorname{Dim} k[\sigma] \mid V(\sigma) \neq(0)\}, \operatorname{Max}_{i}\left\{\operatorname{Dim} S_{i}\right\}\right\} .
$$

(6.3) Theorem. $\operatorname{Dim} \mathscr{M}(A\langle x\rangle)=g=\operatorname{Dim} A\langle x\rangle$.

Proof. We first show $\operatorname{Dim} \mathscr{M}(A\langle x\rangle) \leqslant g$. Let $V \subset \mathscr{M}(A\langle x\rangle)$ be finite dimensional with basis $P_{1}, \ldots, P_{m}$, and let $N$ be the maximum of the degrees of the $P_{i}$. Applying $\Phi$ and the direct sum decomposition of $k\left[x_{1}, \ldots, x_{d}\right] \otimes \mathscr{M}(A)$ we can write

$$
\Phi\left(P_{i}\right)=P_{i}^{(1)}+\cdots+P_{i}^{(n)}+Q_{i} \quad \text { where } P_{i}^{(j)} \in S_{j} \otimes R_{j}
$$

and $Q_{i}=\sum_{\nu} Q_{i, \nu} \otimes a_{\nu}$ where $Q_{i, \nu} \in k\left[X_{1}, \ldots, X_{d}\right]$ and $a_{\nu} \in J$. Using the orthogonality of the distinct $R_{j}$, and hence that of the distinct $S_{j} \otimes R_{j}$, and the nilpotency of $J$, we have

$$
\begin{aligned}
\Phi\left(V^{t}\right) & \subset \operatorname{span}_{k}\left\{\prod_{1 \leqslant i_{j} \leqslant t}\left(P_{i_{j}}^{(1)}+\cdots+P_{i_{j}}^{(n)}+Q_{i_{j}}\right)\right\} \\
& \subset \sum_{i=1}^{n} S_{i} \otimes R_{i}+\sum_{\sigma} M(\sigma) \otimes J
\end{aligned}
$$

for all $t$. Here, $M(\sigma)$ is a finitely generated graded $k[\sigma]$ module. We may take as generators for $M(\sigma)$ all homogeneous summands of all products of the $Q_{i, \nu}$ with at most as many factors as the nilpotency degree of $J$. In particular, for sufficiently large $t, \Phi\left(V^{t}\right)$ is contained in the subspace of $\sum_{i=1}^{n} S_{i} \otimes R_{i}+\sum_{\sigma} M(\sigma) \otimes J$ of terms of degree $\leqslant N t$. The inequality $\operatorname{Dim} \mathscr{M}(A\langle x\rangle) \leqslant g$ now follows from (3.6) and (3.7).

Let $\sigma=\left(i_{1}, \ldots, i_{l}\right)$ satisfy $V(\sigma) \neq(0)$, and $\operatorname{Dim} k[\sigma]=g$. There are positive integers $\alpha_{1}, \ldots, \alpha_{l-1}$ satisfying

$$
R_{i_{1}} J^{\alpha_{1}} R_{i_{2}} J^{\alpha_{2}} \cdots J^{\alpha_{l-1}} R_{i_{l}} \neq(0),
$$


and

$$
R_{i_{1}} J^{\alpha_{1}} \cdots J^{\alpha_{s-1}} R_{i_{s}} J^{\alpha_{s}+1} R_{i_{s+1}} J^{\alpha_{s+1}} \cdots J^{\alpha_{l-1}} R_{l}=(0)
$$

for each $1 \leqslant s \leqslant l-1$. Indeed, take for $\alpha_{1}$ the largest integer for which $R_{i_{1}} J^{\alpha_{1}} R_{i_{2}} J$ $\cdots J R_{i_{l}} \neq(0), \quad \alpha_{2}$ the largest integer for which $R_{i_{1}} J^{\alpha_{1}} R_{i_{2}} J^{\alpha_{2}} R_{i_{3}} J R_{i_{l}} \neq(0)$, etc.

Let $P_{i_{j}}^{\prime} \in S_{i_{j}}$ for each $i_{j} \in \sigma$. By construction, $P_{i_{j}}^{\prime} \otimes 1_{i_{j}}=\tilde{\Phi}\left(\tilde{P}_{i_{j}}\right)$ for some $\tilde{P}_{i_{j}} \in$ $\mathscr{M}(A\langle x\rangle) / \tilde{\mathscr{J}}$, where $1_{i_{j}}$ is the unit in $R_{i_{j}}$. Let $P_{i_{j}} \in \mathscr{M}(A\langle x\rangle)$ be a preimage of $\tilde{P}_{i_{j}}$ and write $\Phi\left(P_{i_{j}}\right)=P_{i_{j}}^{\prime} \otimes 1_{i_{j}}+Q_{j}$ where $Q_{j}=\Sigma Q_{i, \nu} \otimes b_{\nu}$ for some $b_{\nu} \in J$ and $Q_{j, \nu} \in k\left[x_{1}, \ldots, x_{d}\right]$. Consider the product

$$
\begin{aligned}
\left(1 \otimes 1_{i_{1}}\right)\left[P_{i_{1}}^{\prime} \otimes 1_{i_{1}}+Q_{1}\right]\left(1 \otimes 1_{i_{1}}\right)\left(1 \otimes a_{1}\right)\left(1 \otimes 1_{i_{2}}\right) & \cdot\left[P_{i_{2}}^{\prime} \otimes 1_{i_{2}}+Q_{2}\right]\left(1 \otimes 1_{i_{2}}\right)\left(1 \otimes a_{2}\right) \\
& \cdots\left(1 \otimes a_{l-1}\right)\left(1 \otimes 1_{i_{l}}\right)\left[P_{1_{l}}^{\prime} \otimes 1_{i_{l}}+Q_{l}\right]\left(1 \otimes 1_{i_{l}}\right),
\end{aligned}
$$

where $a_{i} \in J^{\alpha_{i}}$ and $1_{i_{1}} a_{1} 1_{i_{2}} a_{i_{2}} \cdots a_{l-1} 1_{i_{l}} \neq 0$. Multiplied out, the only nonzero term is

$$
P_{i_{1}}^{\prime} P_{i_{2}}^{\prime} \cdots P_{i_{l}}^{\prime} \otimes\left(1_{i_{1}} a_{1} l_{i_{2}} a_{2} \cdots a_{l-1} 1_{i_{l}}\right)
$$

by the choice of $\alpha_{1}, \ldots, \alpha_{l-1}$.

By definition, the integral domain $k[\sigma]$ is generated by the subalgebras $S_{i_{j}}$, and $\operatorname{Dim} k[\sigma]$ is equal to the transcendence degree of its field of quotients. In particular, there is a subalgebra of $k[\sigma]$ generated by polynomials $\prod_{1 \leqslant j \leqslant l} P_{i_{j}}^{\prime}, P_{i_{j}}^{\prime} \in S_{i_{j}}$, whose G-K dimension is equal to $\operatorname{Dim} k[\sigma]$. By choosing a subspace of $\mathscr{M}(A\langle x\rangle)$ generated by the $P_{i_{j}}$ corresponding to the $P_{i_{j}}^{\prime}$, and $\mathscr{M}(A)$, a finite type subalgebra of $\mathscr{M}(A\langle x\rangle)$ can be constructed whose $\mathrm{G}-\mathrm{K}$ dimension is at least $\operatorname{Dim} k[\sigma]$.

(6.4) Corollary. For any finite set $X, \operatorname{Dim} A\langle X\rangle=|X| \operatorname{Dim} A\langle x\rangle$.

PROOF. The proof is a modification of the proof of Theorem (6.3) and we use the notation of that proof. We may, without loss of generality, assume that $k$ is algebraically closed.

Let $X=\left\{x_{1}, \ldots, x_{n}\right\}$ and $\{y\}$ be disjoint sets. There are algebra homomorphisms

$$
\begin{gathered}
A\langle X\rangle \rightarrow(A\langle X\rangle)\langle y\rangle \cong A\langle X, y\rangle, \\
\varepsilon: \mathscr{M}(A\langle X\rangle) \rightarrow \mathscr{M}(A\langle X, y\rangle), \\
\Phi_{X}: \mathscr{M}(A\langle X\rangle) \rightarrow k\left[X_{1}, \ldots, X_{d}\right] \otimes \mathscr{M}(A), \\
\Phi_{X, y}: \mathscr{M}(A\langle X, y\rangle) \rightarrow k\left[X_{1}, \ldots, X_{d}, y_{1}, \ldots, y_{d}\right] \otimes \mathscr{M}(A),
\end{gathered}
$$

all of which are graded $k$-algebra monomorphisms. Note that the set $X_{1} \cup \cdots \cup X_{d}$ $\cup\left\{y_{1}, \ldots, y_{d}\right\}$ consists of $d(n+1)$ algebraically independent indeterminates over $k$.

Set

$$
\begin{aligned}
\tilde{J}_{X} & =\Phi_{X}^{-1}\left(k\left[X_{1}, \ldots, X_{d}\right] \otimes J\right), \\
\tilde{J}_{X, y} & =\Phi_{X, y}^{-1}\left(k\left[X_{1}, \ldots, X_{d}, y_{1}, \ldots, y_{d}\right] \otimes J\right),
\end{aligned}
$$


and note that $\tilde{J}_{X}=\varepsilon^{-1}\left(\tilde{J}_{X, y}\right)$. Thus, there is a monomorphism $\tilde{\varepsilon}: \mathscr{M}(A\langle X\rangle) / \tilde{J}_{X} \rightarrow$ $\mathscr{M}(A\langle X, y\rangle) / \tilde{J}_{X, y}$, and a commutative diagram

$$
\begin{aligned}
& \mathscr{M}(A\langle X\rangle) / \tilde{J}_{X} \quad \stackrel{\tilde{\Phi}_{X}}{\rightarrow} \prod_{i} k\left[X_{1}, \ldots, X_{d}\right] \otimes R_{i} \quad \stackrel{\pi_{\alpha}}{\rightarrow} \quad k\left[X_{1}, \ldots, X_{d}\right] \otimes R_{\alpha} \\
& \tilde{\varepsilon} \downarrow \quad \downarrow \prod_{i}\left(\psi \otimes \mathrm{id}_{R_{i}}\right) \quad \downarrow \psi \otimes \operatorname{id}_{R_{\alpha}} \\
& \mathscr{M}(A\langle X, y\rangle) / \tilde{J}_{X, y} \rightarrow \prod_{i} k\left[X_{1}, \ldots, X_{d}, y_{1}, \ldots, y_{d}\right] \otimes R_{i} \stackrel{\tilde{\pi}_{\alpha}}{\rightarrow} k\left[X_{1}, \ldots, X_{d}, y_{1}, \ldots, y_{d}\right] \otimes R_{\alpha}
\end{aligned}
$$

where $\psi: k\left[X_{1}, \ldots, X_{d}\right] \rightarrow k\left[X_{1}, \ldots, X_{d}, y_{1}, \ldots, y_{d}\right]$ is the embedding induced by $X_{i} \rightarrow X_{i}$ for $1 \leqslant i \leqslant d$.

Denote the image of $\pi_{\alpha} \circ \tilde{\Phi}_{X}$ by $S_{X, \alpha}$ and the image of $\pi_{\alpha} \circ \tilde{\Phi}_{X, y}$ by $S_{X, y, \alpha}$. By induction we may assume $\operatorname{Dim} S_{X, \alpha}=n \cdot \operatorname{Dim} S_{\alpha}$, and that a transcendence base for the field of quotients of $S_{X, \alpha}$ over $k$ is given by $\left\{P_{1}\left(x_{11}, \ldots, x_{1 d}\right), \ldots, P_{1}\left(x_{n 1}, \ldots, x_{n d}\right), \ldots, P_{t}\left(x_{11}, \ldots, x_{1 d}\right), \ldots, P_{t}\left(x_{n 1}, \ldots, x_{n d}\right)\right\}$ where $\left\{P_{i}\left(x_{1}, \ldots, x_{d}\right) \mid 1 \leqslant i \leqslant t\right\} \subset S_{\alpha}$ is a transcendence base for the field of quotients of $S_{\alpha}$ as in the proof of (6.3). The polynomials $P_{i}\left(y_{1}, \ldots, y_{d}\right)$ are algebraically independent over $k$ and independent from

$$
\left\{P_{i}\left(x_{j 1}, \ldots, x_{j d}\right) \mid 1 \leqslant i \leqslant t, 1 \leqslant j \leqslant n\right\},
$$

proving that $\operatorname{Dim} S_{X, y, \alpha} \geqslant \operatorname{Dim} S_{X, \alpha}+\operatorname{Dim} S_{\alpha}=(n+1) \operatorname{Dim} S_{\alpha}$. On the other hand, $S_{X, y, \alpha}$ is a homomorphic image of $S_{X, \alpha} \otimes_{k} S_{\alpha}$, from which $\operatorname{Dim} S_{X, y, \alpha} \leqslant \operatorname{Dim} S_{X, \alpha}+$ $\operatorname{Dim} S_{\alpha}$ follows.

Defining $k[\sigma]_{X}$ (resp. $k[\sigma]_{X, y}$ ) to be the subalgebra of $k\left[X_{1}, \ldots, X_{d}\right]$ (resp. the subalgebra of $k\left[X_{1}, \ldots, X_{d}, y_{1}, \ldots, y_{d}\right]$ generated by $\left\{S_{X, \alpha} \mid \alpha \in \sigma\right\}$, (resp. $\left\{S_{X, y, \alpha} \mid \alpha\right.$ $\in \sigma\})$, we obtain $\operatorname{Dim} k[\sigma]_{X, y}=(n+1) \operatorname{Dim} k[\sigma]$. The remainder of the argument goes as in Theorem (6.3).

VII. Concluding remarks. If $B$ is a commutative, associative, unital ring extending a unital subring $A$ with $1_{A}=1_{B}$, then $B$ is a homomorphic image of $A\langle X\rangle$ for some set $X$. Also, any homomorphism of commutative associative unital $R$-algebras, $A \rightarrow B$, where $R$ contains an infinite field, extends naturally to a homomorphism $A\langle X\rangle \rightarrow B\langle X\rangle$. In this case, $A[X] \cong A\langle X\rangle$, and the condition that algebra homomorphisms extend to the algebra of polynomial functions leads to the notion of a nomial homomorphism [11 or 3].

Given a homomorphism $\psi: A \rightarrow B$ of arbitrary $R$-algebras and any set $X, \psi$ together with the set map $X \rightarrow B\langle X\rangle$ given by $x \mapsto x$ induces an algebra homomorphism $\psi_{X, \mathrm{fl}}: A\langle X\rangle_{\mathrm{fl}} \rightarrow B\langle X\rangle$.

(7.1) Definition. A homomorphism $\psi: A \rightarrow B$ of $R$-algebras is said to be nomial provided $\operatorname{In}(A, X) \subset \operatorname{ker}\left(\psi_{X, \mathrm{fl}}\right)$ for all sets $X$.

In other words, $\psi$ is nomial provided $\psi$ extends to a homomorphism $\psi_{X}$ : $A\langle X\rangle \rightarrow B\langle X\rangle$ which agrees with $\psi$ in degree 0 .

(7.2) THEOREM [11]. An injective homomorphism of $R$-algebras $\psi: A \rightarrow B$ is nomial if and only if there is a set $X$ and a surjective homomorphism $\Phi: A\langle X\rangle \rightarrow B$ so that $\left.\Phi\right|_{A}=\psi$. Moreover, $X$ may be taken to be finite if $B$ is finitely generated over the image of $A$. 
If $k$ is an infinite field, then every homomorphism of commutative associative unital $k$-algeras is nomial. It is easily checked that all surjective homomorphisms of arbitrary $R$ algebras are nomial.

If $A$ is commutative, associative, unital and finite dimensional, then $\operatorname{Dim} A=0$ and $\operatorname{Dim} A[x]=1$. Expressed another way, if $k$ is infinite, then $A$ is a nomial extension of $k$ and we have $\operatorname{Dim}-\langle x\rangle$ is constant and equal to 1 on the category of finite dimensional nomial extensions of $k$. This remark is true in greater generality.

(7.3) TheOREM. If $\Phi: A \rightarrow B$ is a nomial extension of finite dimensional associative $k$-algebras then $\operatorname{Dim} A\langle x\rangle=\operatorname{Dim} B\langle x\rangle$.

Proof. It is easily verified that if $L$ is a field extension of $k$, the injection $\mathrm{id}_{L} \otimes \Phi: L \otimes_{k} A \rightarrow L \otimes_{k} B$ is again nomial since, by (4.1), $(L \otimes A)\langle X\rangle \cong L \otimes_{k}$ $A\langle X\rangle$. We may thus assume, without loss of generality, that $k$ is algebraically closed. Furthermore, finite dimensionality of $B$ implies that there is a finite set $X$ and a surjective $\psi: A\langle X\rangle \rightarrow B$ so that $\left.\psi\right|_{A}=\Phi$.

As in $\S I V$, we lift a Wedderburn decomposition $A \cong \prod_{i=1}^{n} R_{i} \oplus J_{A}$ to a Wedderburn decomposition $A\langle X\rangle \cong \prod_{i=1}^{n}\left(k\left[X_{1}^{(i)}, \ldots, X_{d_{i}}^{(i)}\right] \otimes R_{i}\right) \oplus \mathscr{J}$. Denote by $S_{X}$ the separable subalgebra $\prod_{i} k\left[X_{1}^{(i)}, \ldots, X_{d_{i}}^{(i)}\right] \otimes R_{i}$, and identify $S_{X}$ and $\mathscr{J}$ with their image is in $A\langle X\rangle$, obtaining a homomorphism, denoted $\Psi$, from $S_{X} \oplus \mathscr{J}$ onto $B$. Note that $\Psi(\mathscr{J})$ is a nilpotent ideal in $B$, so that $\Psi$ induces a surjection $S_{X} \rightarrow B / J_{B}$ where $J_{B}$ is the radical of $B$.

$S_{X}$ has a basis of the form $\left\{P_{\alpha}\left(X_{1}^{(i)}, \ldots, X_{d_{i}}^{(i)}\right) \otimes e_{i}^{(j)} \mid 1 \leqslant i \leqslant n, 1 \leqslant j \leqslant d_{i}\right\}$ and $\mathscr{J}$ has a basis $\left\{Q_{\beta}\left(X_{1}^{(1)}, \ldots, X_{d_{n}}^{(n)}, y_{1}, \ldots, y_{n}\right) \otimes f^{i}\right\}$ where $\left\{e_{(i)}^{j} \mid 1 \leqslant j \leqslant d_{i}\right\}$ is a basis for $R_{i}$ and $\left\{f^{i} \mid 1 \leqslant i \leqslant r\right\}$ a basis for $J_{A}$. Together with the fact that $\left.\Psi\right|_{A}=\Phi$, and $\Phi$ is injective, we obtain $B \cong \Psi\left(S_{X}\right) \oplus \Psi(J)$. Setting $\Psi_{i}=$ $\left.\Psi\right|_{k\left[X_{1}^{(i)}, \ldots, X_{d_{i}}^{(i)}\right] \otimes R_{i}}$, a Wedderburn decomposition of $B$ is given by $B \cong S_{B} \oplus J_{B}$ where $S_{B}$ is a homomorphic image of $S_{X}$. Indeed, $S_{B} \cong \prod_{i=1}^{n} \prod_{\alpha=1}^{n_{i}} R_{i, \alpha}$ where $\prod_{\alpha=1}^{n_{i}} R_{i, \alpha} \cong\left(\mathrm{im} \Psi_{i}\right) / \operatorname{rad}\left(\mathrm{im} \Psi_{i}\right)$, and $R_{i, \alpha} \cong R_{i}$. Therefore, $\Psi$ decomposes as $\Psi=$ $\left(\Pi \Psi_{i}\right) \oplus \Psi \mid \mathscr{J}$ with

$$
\Psi_{i}: k\left[X_{i}^{(1)}, \ldots, X_{d_{i}}^{(i)}\right] \otimes R_{i} \rightarrow\left(\prod_{\alpha=1}^{n_{i}} R_{i, \alpha}\right) \oplus J_{B} .
$$

The kernel of $\Psi_{i}$ has the form $\left(L_{1} \cap \cdots \cap L_{n_{i}}\right) \otimes R_{i}$ where the $L_{\alpha}$ are ideals in $k\left[X_{1}^{(i)}, \ldots, X_{d_{i}}^{(i)}\right]$ primary to distinct maximal ideals $M_{\alpha}$. Thus

$$
\begin{aligned}
\operatorname{im} \Psi_{i} & \cong \frac{k\left[X_{1}^{(i)}, \ldots, X_{d_{i}}^{(i)}\right]}{\bigcap L_{\alpha}} \otimes R_{i} \cong \prod_{\alpha} \frac{k\left[X_{1}^{(i)}, \ldots, X_{d_{i}}^{(i)}\right]}{L_{\alpha}} \otimes R_{i} \\
& \cong \prod_{\alpha} \frac{k\left[X_{1}^{(i)}, \ldots, X_{d_{i}}^{(i)}\right]}{M_{\alpha}} \oplus \frac{M_{\alpha}}{L_{\alpha}} \otimes R_{i} .
\end{aligned}
$$

Since $M_{\alpha} / L_{\alpha} \cong \operatorname{rad} k\left[X_{1}^{(i)}, \ldots, X_{d_{i}}^{(i)}\right]$, we obtain

$$
\operatorname{rad}\left(\operatorname{im} \Psi_{i}\right) \cong \prod_{\alpha=i}^{n_{i}}\left(M_{\alpha} / L_{\alpha}\right) \otimes R_{i} .
$$


A complete description of $B$ is given by

$$
\begin{gathered}
B \cong \prod_{i}\left(\frac{\prod_{\alpha} k\left[X_{i}^{(i)}, \ldots, X_{d_{i}}^{(i)}\right]}{M_{i, \alpha}} \otimes R_{i}\right) \oplus\left(\bigoplus_{i}\left(\bigoplus_{\alpha} \frac{M_{i, \alpha}}{L_{i, \alpha}} \otimes R_{i}\right)\right) \oplus \Psi(\mathscr{J}), \\
S_{B} \cong \prod_{i} \frac{\prod_{\alpha} k\left[X_{i}^{(i)}, \ldots, X_{d_{i}}^{(i)}\right]}{M_{i, \alpha}} \otimes R_{i}, \\
J_{B} \cong \bigoplus_{i} \bigoplus_{\alpha}\left(\frac{M_{i, \alpha}}{L_{i, \alpha}} \otimes R_{i}\right) \oplus \Psi(\mathscr{J}) .
\end{gathered}
$$

By Theorem (5.1) and the observation that $R_{i, \alpha} \cong R_{i}$ for each $\alpha, \operatorname{Dim} A\langle x\rangle=$ $\operatorname{Dim} B\langle x\rangle$ will follow if it is shown that every nonzero subspace $R_{i_{1}, \alpha_{1}} J_{B} R_{i_{2}, \alpha_{2}} J_{B}$ $\cdots J_{B} R_{i_{m}, \alpha_{m}}$ of $B$ with distinct $R_{i_{j}}, \alpha_{i_{j}}$ has all $i_{j}$ distinct. For this, it suffices to show that $R_{i_{\alpha}} J_{B} R_{i_{\beta}}=(0)$ for $\alpha \neq \beta$.

The orthogonality of the distinct $R_{i}$ in $A$, and the distinct $k\left[X_{1}^{(i)}, \ldots, X_{d}^{(i)}\right] / L_{\alpha}$ in $A\langle X\rangle$, yields

$$
(0)=\left[\frac{k\left[X_{1}^{(i)}, \ldots, X_{d_{i}}^{(i)}\right]}{M_{i, \alpha}} \otimes R_{i}\right]\left[\frac{M_{j, \gamma}}{L_{j, \gamma}} \otimes R_{j}\right]\left[\frac{k\left[X_{1}^{(i)}, \ldots, X_{d_{i}}^{(i)}\right]}{M_{i, \beta}} \otimes R_{i}\right] .
$$

We are thus reduced to showing $R_{i, \alpha} \Psi(\mathscr{J}) R_{i, \beta}=(0)$ for $\alpha \neq \beta$. To that end, let

$$
\begin{aligned}
& P=\sum P_{j}\left(X_{1}^{(i)}, \ldots, X_{d_{i}}^{(i)} \otimes e_{(i)}^{j} \in \Psi_{i}^{-1}\left(R_{i, \alpha}\right),\right. \\
& Q=\sum Q_{k}\left(X_{1}^{(1)}, \ldots, X_{d_{n}}^{(n)}, y_{1}, \ldots, y_{s}\right) \otimes f^{k} \in \mathscr{J}, \\
& T=\sum T_{l}\left(X_{1}^{(i)}, \ldots, X_{d_{i}}^{(i)}\right) \otimes e_{(i)}^{l} \in \Psi_{i}^{-1}\left(R_{i, \beta}\right) .
\end{aligned}
$$

Then

$$
\begin{aligned}
\Psi(P Q T) & =\Psi\left(\sum_{j, k, l} P_{j} Q_{k} T_{l} \otimes e_{(i)}^{j} f^{k} e_{(i)}^{l}\right) \\
& \left.=\sum_{j, k, l} \bar{P}_{j} \bar{T}_{l} \bar{Q}_{k} \otimes e_{(i)}^{j} f^{k} e_{(i)}^{l}\right)
\end{aligned}
$$

where $\bar{P}_{j}$ and $\bar{T}_{l}$ are the images of $P_{j}$ and $T_{l}$ in the orthogonal rings $k\left[X_{1}^{(i)}, \ldots, X_{d}^{(i)}\right] / M_{i, \alpha}, k\left[X_{1}^{(i)}, \ldots, X_{d}^{(i)}\right] / M_{i, \beta}$. In particular, $\Psi(P Q T)=0$.

It is conjectured that Theorem (7.3) holds in the full generality of the category of finite dimensional, not necessarily associative, algebras with nomial homomorphisms.

\section{REFERENCES}

1. W. Borho and H. Kraft, Über die Gelfand-Kirillov Dimension, Math. Ann. 220 (1976), 1-24.

2. C. Faith, Algebra, Springer-Verlag, 1976.

3. D. R. Finston, The algebra of polynomial functions on a non-associative algebra, Thesis, U.C.S.D., 1983.

4. $1597-1626$

, Separable polynomials over finite dimensional algebras, Comm. Algebra 13 (1985),

5. __ On multiplication algebras, Trans. Amer. Math. Soc. 293 (1986), 807-818.

6. N. Jacobson, The theory of rings, Math. Surveys, no. 2, Amer. Math. Soc., Providence, R. I., 1943. 
7. A. Joseph and L. W. Small, An additivity principle for Goldie rank, Israel J. Math. 31 (1978), $105-114$.

8. G. R. Krause and T. H. Lenagan, Growth of algebras and Gelfand-Kirillov dimension, Pitman, 1985.

9. M. Lorenz and L. W. Small, On the Gelfand-Kirillov dimension of noetherian P. I. algebras, Contemp. Math., vol. 13, Amer. Math. Soc., Providence, R. I., 1982, pp. 199-205.

10. H. Röhrl, On the zeros of polynomials over arbitrary finite dimensional algebras, Manuscripta Math. 25 (1978), 359-390.

11. The algebra of polynomial functions with coefficients in a nonassociative algebra, Seminar berichte Fernuniversität Hagen 6 (1980) and 8 (1981).

Department of Mathematical Sciences, Virginia Commonwealth University, Richmond, VIRGINIA 23284 - 0001 